\title{
Nonlinear interfacial dynamics in stratified multilayer channel flows
}

\author{
E.S. P A P A E T T Y M I U, D.T. PAPA GEORG I OU \\ AND G.A. PAVLIOTIS
}

Department of Mathematics, Imperial College London, London SW7 2AZ, UK.

(Received 4 July 2013)

The dynamics of viscous immiscible pressure-driven multilayer flows in channels are investigated using a combination of modelling, analysis and numerical computations. More specifically the particular system of three stratified layers with two internal fluid-fluid interfaces is considered in detail in order to identify the nonlinear mechanisms involved due to multiple fluid surface interactions. The approach adopted is analytical/asymptotic and is valid for interfacial waves that are long compared to the channel height or individual undisturbed liquid layer thicknesses. This leads to a coupled system of fully nonlinear partial differential equations of Benney-type that contain a small slenderness parameter that cannot be scaled out of the problem. This system is in turn used to develop a consistent coupled system of weakly nonlinear evolution equations, and it is shown that this is possible only if the underlying base-flow and fluid parameters satisfy certain conditions that enable a synchronous Galilean transformation to be performed at leading order. Two distinct canonical cases (all terms in the equations are of the same order) are identified in the absence and presence of inertia, respectively. The resulting systems incorporate all the active physical mechanisms at Reynolds numbers that are not large, namely, nonlinearities, inertia-induced instabilities (at non-zero Reynolds number) and surface tension stabilisation of sufficiently short waves. The coupled system supports several instabilities that are not found in single long-wave equations including, transitional instabilities due to a change of type of the flux nonlinearity from hyperbolic to elliptic, kinematic instabilities due to the presence of complex eigenvalues in the linearised advection matrix leading to a resonance between the interfaces, and the possibility of long-wave instabilities induced by an interaction between the flux function of the system and the surface tension terms. All these instabilities are followed into the nonlinear regime by carrying out extensive numerical simulations using spectral methods on periodic domains. It is established that instabilities leading to coherent structures in the form of nonlinear travelling waves are possible even at zero Reynolds number, in contrast to single interface (two-layer) systems; in addition, even in parameter regimes where the flow is linearly stable, the coupling of the flux functions and their hyperbolic-elliptic transitions lead to coherent structures for initial disturbances above a threshold value. When inertia is present an additional short-wave instability enters and the systems become general coupled Kuramoto-Sivashinsky type equations. Extensive numerical experiments indicate a rich landscape of dynamical behaviour including nonlinear travelling waves, time-periodic travelling states and chaotic dynamics. It is also established that it is possible to regularise the chaotic dynamics into travelling wave pulses by enhancing the inertialess instabilities through the advective terms. Such phenomena may be of importance in mixing, mass and heat-transfer applications. 


\section{Introduction}

Multilayer channel flows, either pressure or gravity driven, have received considerable attention due to their rich dynamical behaviour and their enormous range of scientific and industrial applications. The advancement of novel technologies based on microfluidic platforms (e.g. lab-on-chip systems) has created numerous of applications involving multilayer flows in microchannels. At the same time, the accurate control and manipulation of multilayer microscale flows has become increasingly popular in modern biomedical and other applications. Examples include techniques for concentrating leukocytes from whole blood samples (see SooHoo \& Walker 2009), integrated lab-on-chip systems (see Beebe et al. 2002; Figeys \& Pinto 2000; Hibara et al. 2001; Surmeian et al. 2002), and the use of microfluidic devices in food engineering (Skurtys \& Aguilera 2008). Potential applications in MEMS (micro-electro-mechanical) devices in the aerospace industry have been suggested, such as microthrusters that can propel small scale spacecrafts and satellites - see (Polsin \& Choueiri 2002). Furthermore, multilayer flows are also encountered in oil production and transportation in the petrochemical industry (Lovick \& Angeli 2004), and in processing industries including multilayer coextrusion processes (Nordberg \& Winter 1988) and multilayer coating processes (Weinstein \& Ruschak 2004). One of the main objectives of the present study is to understand the stability of such flows and in particular to follow instabilities into the nonlinear regime in order to explore and quantify the different types of underlying nonlinear phenomena that are crucial in applications.

The linear stability of two-layer flows in channels (either plane Couette or Poiseuille), where only one interface is present and separates fluids of different viscosities and densities, was first studied by Yih (1967). Yih considered the long-wave limit and showed that there exists an unstable mode associated with the jump in viscosity across the interface (termed an interfacial mode) at arbitrarily small but non-zero values of the Reynolds number. Yih's study suggests a simple "rule of thumb" in finding linearly stable flows, at least at small Reynolds numbers: arrange the less viscous fluid in the thinner of the two layers to stabilise long-waves, and include sufficient amounts of surface tension to stabilise short waves. Experimental observations and numerical calculations of the full linear eigenvalue problem support this finding, and interestingly Rayleigh-Taylor unstable arrangements (heavier fluid on top) can also be stabilised this way. For a detailed discussion of the stability of two-layer Couette-Poiseuille flow, along with numerous references on analysis, computations and experiments, the reader is referred to the monograph by Joseph \& Renardy (1991). Later studies by Tilley et al. (1994a, b), consider the linear and nonlinear stability of two-layer flow in an inclined channel allowing for counter-flowing flows. Given the success of long-wave linear theories in horizontal channels, analyses were carried out to derive weakly nonlinear long-wave models described by the Kuramoto-Sivashinsky equation - see for example Hooper \& Grimshaw (1985) and Shlang \& Sivashinsky (1985).

The present work is concerned with instabilities and nonlinear dynamics in three-layer flows in an inclined plane channel, where two fluid-fluid interfaces are now present and can interact among themselves and with the solid boundaries; the set-up is a complex but amenable system allowing an extended study of nonlinear fluid-surface interactions. Multilayer flows with several internal interfaces behave quite differently from single interface (or single surface) flows in several important aspects. Multilayer flows depend on additional physical parameters and perhaps more importantly support a resonance mechanism between the interacting interfaces (or the interfaces and the free surface in the case of open flows). As a result, such systems (both closed and open) support instabilities that are not seen in two-layer flows. It has been established that two-layer 
flows in inclined or pressure-driven channels and single-layer free-surface flows down inclined planes, require fluid inertia for destabilisation, at least when the inclination to the horizontal is less than ninety degrees (see Chen 1995; Beniamin 1957; Yih 1963). However, in the case of two-layer free-surface flows, Kao (1968). Loewenherz \& Lawrence (1989) and Chen (1993) showed that when the less viscous fluid is adjacent to the wall, then a long-wave instability can appear in the absence of inertia (zero Reynolds number); this instability has been termed inertialess instability. Chen (1993) argues that the instability arises from an interaction between the free surface and the interface, while an interpretation of the underlying mechanism has been given recently by Gao \& Lu (2008). An analogous linear stability study was undertaken by Li (1969), for Couette flow of three superposed fluids of different viscosities; it was shown that the flow can become unstable in the long-wavelength limit for certain values of the depth and viscosity ratio due to resonance between the interfaces, something that does not happen if the additional interface is not present. A weakly nonlinear study of three-layer Poiseuille flow was considered by Kliakhandler \& Sivashinskv (1995), who derived a system of weakly nonlinear evolution equations corresponding to Li's instability, and observed two new kinds of long-wavelength inertialess instabilities; one of entirely kinematic nature known as the "alpha"-effect, and an additional surface-tension-induced instability. For a recent review of multilayer instabilities in flows in channels and films, see (Pozrikidis 2004).

Here we focus on the long-wavelength instabilities mentioned previously, both in the absence and presence of inertia. The novel feature of multilayer flows is the introduction of kinematic instabilities that are absent in single-interface problems. As a result, careful asymptotic analysis must be performed to arrive at canonical lower-dimension systems not involving a small arbitrary parameter, and we will carry this out in order to identify the correct models (we note that the weakly nonlinear models suggested in (Kliakhandler \& Sivashinskv 1995) and (Kliakhandler \& Sivashinskv 1996) were not appropriately derived as will be explained later). The difficulty lies in removing the leading order advective terms present, to ensure that the evolution equation does not contain an arbitrary small parameter. In general, for flows involving a single interface (or free surface) a single evolution equation is found and an appropriate Galilean transformation can be used to obtain an asymptotically correct evolution equation (see for example Papageorgiou et al. (1990) for the derivation of equations in core-annular flows). However, in the case of multilayer flows we obtain a system of evolution equations (as in (Kliakhandler \& Sivashinsky 1995), for example), necessitating that the matrix of the advective terms should be diagonal with equal eigenvalues to enable an appropriate Galilean transformation to be performed. With a Galilean transformation identified, an asymptotic de-tuning of the parameters (in the present physical problem there are eight such parameters, two of each of the following ratios: viscosity, density, surface tension, undisturbed layer thickness), along with nonlinear balances, provides the rich canonical systems of equations that form the basis of our analytical and computational studies. This Galilean transformation requirement can be satisfied in the case of a closed flow inside a channel, but for the open flow of a falling film multilayer system we have verified that a similar transformation cannot be performed. Consequently, the weakly nonlinear models derived in (Kliakhandler 1999) for multilayer thin films down an inclined plane require additional study. An extensive linear stability study of such multilayer falling film flows, showing the existence of long-wave and inertialess unstable modes has been carried out by (Jiang et al. 2005). These authors also performed experiments on aqueous gelatin systems to confirm the existence of three-layer inertialess instability.

Finally, we would like to draw attention to the kind of nonlinearities involved in our weakly nonlinear models and to make a connection with several other applica- 
tions that potentially share the phenomena and mathematical structures elucidated here. The nonlinearities are quadratic flux functions (the equations are quasilinear) and for certain initial conditions and physical parameters of the problem, they can themselves be a source of inertialess instability when the Jacobian matrix of the flux function possesses complex conjugate eigenvalues (the nonlinearities in the partial differential equations (PDEs) are of mixed hyperbolic-elliptic type). This feature has been studied in the context of systems of conservation laws arising in fluid dynamics problems such as stratified flows (Milewski et al. 2004), (Chumakova et al. 2009), jet flows (Papageorgiou \& Orellana 1998), steady transonic flows (Cole \& Cook 1996), magnetofluid dynamics (Kogan 1961), in fluids of van der Waals type (Slemrod 1983), and in three-phase convection-driven flow in porous media modelling fluid flows in petroleum reservoirs (Bell et al. 1986). Additionally, these quadratic nonlinearities can be derived as an approximation of more general flux functions in the neighborhood of isolated singular points in the state space (Schaeffer \& Shearer 1987). Furthermore, even when the Jacobian matrix possesses real and distinct eigenvalues, the nonlinearities can introduce instability into the system by interacting with the other terms present, e.g. surface tension. Such kinds of instabilities were found by Majda \& Pego (1985) who studied admissible viscosity matrices for strictly hyperbolic conservation laws. The present study develops PDEs that admit all the mathematical and physical features mentioned above, and additionally provides physically meaningful systems with dissipation matrices of fourth-order.

The remainder of the paper is organised as follows. Section 2 provides the governing equations and boundary conditions for the three-phase flow under consideration and also contains the derivation of the coupled fully nonlinear system of long-wave equations that describe the spatio-temporal evolution of the two interfaces. This coupled system contains a small parameter $\delta$ (proportional to the ratio of the channel depth and a typical perturbation wavelength), and in Section 3 we describe the asymptotically correct derivation of canonical weakly nonlinear models. This is done for two physically distinct situations, a symmetric case where inertial effects are felt at higher order, and a nonsymmetric case with inertia terms entering in the form of short-wavelength instabilities (there is of course short-wavelength cutoff due to surface tension effects ensuring that the models are well-posed). Section 3 provides also a discussion of several instability conditions that can affect the evolving solutions including hyperbolic-elliptic transitions as well as Majda-Pego instability. In Section 4 we undertake a computational investigation of solutions to the canonical problems and utilise highly accurate and stable implicitexplicit time integrators with spectral spatial discretisations to investigate solutions at large times. Solutions are presented to quantify all the underlying instability mechanisms and to follow them into their fully nonlinear regime. In Section 5 we make some concluding remarks.

\section{Mathematical formulation and derivation of fully nonlinear long-wave systems}

Consider a two-dimensional multilayer flow driven by gravity and an imposed pressure gradient along an inclined channel of height $d$ as depicted in figure 1. Three immiscible, incompressible Newtonian liquids of constant densities $\rho_{i}$ and viscosities $\mu_{i}, i=1,2,3$, flow together in the channel which is inclined at an angle $\theta$ to the horizontal as shown in the figure. A Cartesian frame of reference $(x, y)$ is adopted with $x$ measuring distances down the channel and $y$ the distance perpendicular to it. The disturbed interface between fluids 1 and 2 is denoted by $y=h_{2}(x, t)$ while that between fluid 2 and 3 is $y=h_{3}(x, t)$ 
(the corresponding undisturbed interfaces are $\mathrm{H}_{2}$ and $H_{3}$, respectively and here $\mathrm{H}_{2}>$ $\left.H_{3}>0\right)$. Consequently, fluids 1,2 and 3 occupy the evolving regions $h_{2}(x, t)<y<d$, $h_{3}(x, t)<y<h_{2}(x, t)$ and $0<y<h_{3}(x, t)$, respectively. Typically a pressure gradient acts in the $x$-direction and gravitational forces are also accounted for through the force g shown in the figure. In addition, surface tension is present with values $\sigma_{2}$ and $\sigma_{3}$ on interface $h_{2}$ and $h_{3}$, respectively.

The governing equations are the incompressible Navier-Stokes equations in each fluid region along with appropriate boundary conditions across $h_{2,3}(x, t)$. Letting the velocity field in each layer $i$ be $\boldsymbol{u}_{i}=\left(u_{i}, v_{i}\right)^{\top}$ (the superscript $\top$ denotes transpose) and the corresponding pressure be $P_{i}$, the momentum and continuity equations in each layer $i=1,2,3$, are

$$
\begin{gathered}
\rho_{i}\left(\partial_{t} \boldsymbol{u}_{i}+\left(\boldsymbol{u}_{i} \cdot \nabla\right) \boldsymbol{u}_{i}\right)=-\nabla P_{i}+\mu_{i} \nabla^{2} \boldsymbol{u}_{i}+\rho_{i} \mathbf{g} \\
\boldsymbol{\nabla} \cdot \boldsymbol{u}_{i}=0 .
\end{gathered}
$$

There are several boundary conditions to impose at liquid-solid and liquid-liquid surfaces. These are no-slip conditions at liquid-solid and continuity of velocities at liquid-liquid surfaces, yielding

$$
\begin{gathered}
u_{1}=0 \quad, \quad v_{1}=0 \quad \text { at } y=d, \\
u_{3}=0 \quad, \quad v_{3}=0 \quad \text { at } y=0, \\
u_{i-1}=u_{i} \quad, \quad v_{i-1}=v_{i} \quad \text { at } y=h_{i}, \quad i=2,3 .
\end{gathered}
$$

In addition we need to impose continuity of stresses at liquid-liquid interfaces, and resolving these into their tangential and normal components at $y=h_{2}, h_{3}$, yields

$$
\begin{gathered}
\boldsymbol{t}_{i}^{\top} \cdot \boldsymbol{T}_{i-1} \cdot \boldsymbol{n}_{i}=\boldsymbol{t}_{i}^{\top} \cdot \boldsymbol{T}_{i} \cdot \boldsymbol{n}_{i}, \quad i=2,3, \\
\boldsymbol{n}_{i}^{\top} \cdot \boldsymbol{T}_{i-1} \cdot \boldsymbol{n}_{i}=\boldsymbol{n}_{i}^{\top} \cdot \boldsymbol{T}_{i} \cdot \boldsymbol{n}_{i}-\sigma_{i} \kappa_{i}, \quad i=2,3 .
\end{gathered}
$$

Here $\boldsymbol{T}_{i}$ is the the stress tensor in region $i$ given by

$$
\boldsymbol{T}_{i}=\left[\begin{array}{cc}
-P_{i}+2 \mu_{i} u_{i x} & \mu_{i}\left(u_{i y}+v_{i x}\right) \\
\mu_{i}\left(u_{i y}+v_{i x}\right) & -P_{i}+2 \mu_{i} v_{i y}
\end{array}\right],
$$

(subscripts $x, y$ denote partial derivatives), the outward-pointing unit normal is $\boldsymbol{n}_{i}=$ $\left(-h_{i x}, 1\right)^{\top} / \sqrt{1+h_{i x}^{2}}$, the corresponding unit tangent is $\boldsymbol{t}_{i}=\left(1, h_{i x}\right)^{\top} / \sqrt{1+h_{i x}^{2}}$ and the curvature $\kappa_{i}$ at interface $i$ is $\kappa_{i}=h_{i x x} /\left(1+h_{i x}^{2}\right)^{3 / 2}$. After some algebra, the stressbalances (2.6) and (2.7) at $y=h_{i}, i=2,3$, can be written in expanded form as

$$
\begin{gathered}
\mu_{(i-1)} v_{(i-1) x}-\mu_{i} v_{i x}+\mu_{(i-1)} u_{(i-1) y}-\mu_{i} u_{i y}=-\frac{4 h_{i x}}{1-h_{i x}^{2}}\left(\mu_{(i-1)} v_{(i-1) y}-\mu_{i} v_{i y}\right) \\
P_{(i-1)}-P_{i}-2 \frac{1+h_{i x}^{2}}{1-h_{i x}^{2}}\left(\mu_{(i-1)} v_{(i-1) y}-\mu_{i} v_{i y}\right)=\sigma_{i} \kappa_{i}
\end{gathered}
$$

Finally, we have kinematic boundary conditions at $h_{2}, h_{3}$ and these read

$$
h_{i t}+u_{i} h_{i x}-v_{i}=0, \quad i=2,3 .
$$

In addition to conditions at the walls and interfaces we also need to specify conditions 


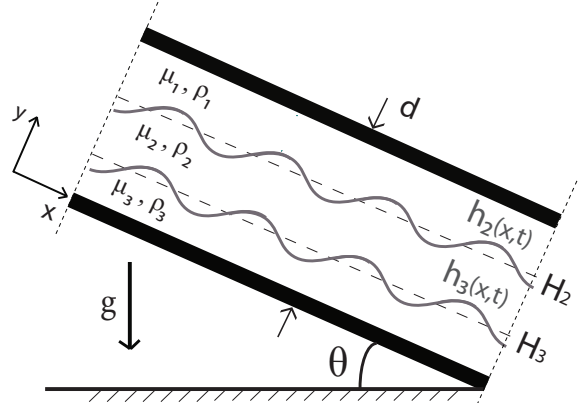

FIGURE 1. Schematic of a three-layer flow down an inclined channel. The undisturbed interfaces are flat and given by $y=H_{2}$ and $H_{3}$.

in the $x$-direction. Throughout this study we will impose periodic boundary conditions along the channel.

In order to complete the mathematical statement of the physical problem, an additional condition needs to be imposed concerning the overall flow rate. This is given by

$$
\int_{0}^{h_{3}} u_{3} d y+\int_{h_{3}}^{h_{2}} u_{2} d y+\int_{h_{2}}^{d} u_{1} d y=Q,
$$

where $Q$ is a constant; (2.12) is imposed by the continuity of the velocities at the interfaces, as will be explained later. In this work, we adopt the scenario where the overall flow rate is fixed and, for the sake of simplicity, equal to unity in dimensionless terms (see below), suggesting that the pressure gradients in each layer will be determined as part of the solution. Alternatively, one can impose a fixed overall pressure gradient in the streamwise direction, in which case the overall flow rate will be determined as part of the solution. Anologous conditions have been adopted by Tillev et al. $(1994 b)$ in their study of two-layer flows, as well as Kliakhandler \& Sivashinsky (1995) in multilayer flows.

\subsection{Dimensionless equations}

We non-dimensionalize the equations using $d$ for lengths, $\bar{U}=\left(g d^{2} \rho_{1} \sin \theta\right) /\left(2 \mu_{1}\right)$ for velocities, $d / \bar{U}$ for time, the viscous pressure scale $\left(2 \mu_{1} U\right) / d$ for pressures, and $\bar{U} d$ for the overall flow rate. Furthermore, we define the ratio of viscosities and densities by $m_{i}=\mu_{i} / \mu_{1}, r_{i}=\rho_{i} / \rho_{1}$, respectively, and the Reynolds and capillary numbers $R e$ and $C$, representing the ratios of inertial to viscous forces, and viscous to capillary forces, respectively, that are given by

$$
R e=\frac{\bar{U} \rho_{1} d}{\mu_{1}}=\frac{g d^{3} \rho_{1}^{2} \sin \theta}{2 \mu_{1}^{2}}, \quad C_{i}=\frac{2 \bar{U} \mu_{1}}{\sigma_{i}}=\frac{g d^{2} \rho_{1} \sin \theta}{\sigma_{i}} .
$$

Consequently, the Navier-Stokes equations become, for $i=1,2,3$,

$$
\begin{gathered}
\operatorname{Re}\left(u_{i t}+u_{i} u_{i x}+v_{i} u_{i y}\right)=-\frac{2}{r_{i}} P_{i x}+\frac{m_{i}}{r_{i}}\left(u_{i x x}+u_{i y y}\right)+2, \\
R e\left(v_{i t}+u_{i} v_{i x}+v_{i} v_{i y}\right)=-\frac{2}{r_{i}} P_{i y}+\frac{m_{i}}{r_{i}}\left(v_{i x x}+v_{i y y}\right)-2 \cot \theta, \\
u_{i x}+v_{i y}=0 .
\end{gathered}
$$


The boundary conditions (2.9), (2.10) and (2.11) at the $i$ th interface $y=h_{i}, i=2,3$, can be written as

$$
\begin{gathered}
m_{(i-1)} v_{(i-1) x}-m_{i} v_{i x}+m_{(i-1)} u_{(i-1) y}-m_{i} u_{i y}=-\frac{4 h_{i x}}{1-h_{i x}^{2}}\left(m_{(i-1)} v_{(i-1) y}-m_{i} v_{i y}\right) \\
P_{(i-1)}-P_{i}-\frac{1+h_{i x}^{2}}{1-h_{i x}^{2}}\left(m_{(i-1)} v_{(i-1) y}-m_{i} v_{i y}\right)=\frac{h_{i x x}}{C_{i}\left(1+h_{i x}^{2}\right)^{3 / 2}}, \\
h_{i t}+u_{i} h_{i x}-v_{i}=0 .
\end{gathered}
$$

Finally, condition (2.12) for the overall flow rate becomes

$$
\int_{0}^{h_{3}} u_{3} d y+\int_{h_{3}}^{h_{2}} u_{2} d y+\int_{h_{2}}^{1} u_{1} d y=1 .
$$

Note that for simplicity we have used the same symbols to represent dimensionless and dimensional dependent and independent variables.

\subsection{Steady-states}

Steady-states emerge by making the interfaces flat, $h_{2}=H_{2}, h_{3}=H_{3}$, and setting $v_{i}=0$, for $i=1,2,3$. In addition the driving pressure gradient $\bar{P}_{i x}$ is constant and is denoted by $\bar{P}_{x}$. It follows from the momentum equation (2.15) that the hydrostatic pressure gradient in the $y$-direction is constant in each layer, and is given by

$$
\bar{P}_{i y}=-r_{i} \cot \theta \text {. }
$$

Finally, the steady horizontal velocity profile in each layer is parabolic in the $y$-direction and a function of $y$ alone,

$$
\frac{m_{i}}{r_{i}} \bar{u}_{i}=\left(-1+\frac{\bar{P}_{x}}{r_{i}}\right) y^{2}+c_{2 i} y+c_{3 i} .
$$

The seven constants $\bar{P}_{x}, c_{2 i}$ and $c_{3 i}, i=1,2,3$ can be fully determined by solving the system (2.22) along with the stress balance equation at the $i$ th interface (2.17), the condition for the flow rate (2.20) and the no-slip conditions for the velocities (2.3) - (2.5) (these remain unaltered when non-dimensionalized, but the wall is now at $y=1$ ). The algebra is cumbersome and was carried out by a computer algebra package.

\subsection{Long-wave asymptotic analysis}

The mathematical model described in section 2.1 (equations (2.14)-(2.20) , when supplemented with initial conditions constitutes a formidable multiphase nonlinear movingboundary problem. To make analytical progress we proceed asymptotically and seek a system of nonlinear evolution equations that can describe the flow when interfacial undulations are long compared to the channel height. More precisely we assume that the typical interfacial deformation wavelength $\lambda$ is large compared to the channel height $d$, i.e. $\delta=d / \lambda<<1$, providing scale separation. We write the flow components $u_{i}, v_{i}, P_{i}$ as the undistributed steady state (see equations (2.21) and (2.22) plus a disturbance of arbitrary size

$$
u_{i}=\bar{u}_{i}+\tilde{u}_{i}, \quad v_{i}=\tilde{v}_{i}, \quad P_{i}=\bar{P}_{i}+\tilde{P}_{i}
$$


The scale separation dictates the following canonical change of variables

$$
\begin{gathered}
x \rightarrow \frac{\xi}{\delta}, \quad y \rightarrow y, \quad t \rightarrow \frac{\tau}{\delta}, \\
\tilde{u}_{i}=\tilde{u}_{i}, \quad \tilde{v}_{i}=\delta \tilde{w}_{i}, \quad \tilde{P}_{i}=\frac{\tilde{p}_{i}}{\delta},
\end{gathered}
$$

where the new quantities $\xi, \tau, \tilde{w}_{i}, \tilde{p}_{i}$ are of order one, while other unscaled variables retain their original definitions. The scaling for $v_{i}$ follows from the continuity equation (2.16) and (2.24), while the order $1 / \delta$ pressures are required in order to retain a flow driven by the horizontal pressure gradient to leading order. Changing variables in the governing equations (2.14)-(2.16) we obtain, for $i=1,2,3$,

$$
\begin{gathered}
\delta R e\left(\tilde{u}_{i \tau}+\bar{u}_{i} \tilde{u}_{i \xi}+\tilde{u}_{i} \tilde{u}_{i \xi}+\tilde{w}_{i} \bar{u}_{i y}+\tilde{w}_{i} \tilde{u}_{i y}\right)=-\frac{2 \tilde{p}_{i \xi}}{r_{i}}+\frac{m_{i}}{r_{i}}\left(\delta^{2} \tilde{u}_{i \xi \xi}+\tilde{u}_{i y y}\right), \\
\delta^{2} \operatorname{Re}\left(\tilde{w}_{i \tau}+\bar{u}_{i} \tilde{w}_{i \xi}+\tilde{u}_{i} \tilde{w}_{i \xi}+\tilde{w}_{i} \tilde{w}_{i y}\right)=-\frac{2 \tilde{p}_{i y}}{\delta r_{i}}+\frac{m_{i}}{r_{i}}\left(\delta^{3} \tilde{w}_{i \xi \xi}+\delta \tilde{w}_{i y y}\right), \\
\tilde{u}_{i \xi}+\tilde{w}_{i y}=0 .
\end{gathered}
$$

The interfacial boundary conditions (2.17)-(2.19) (representing tangential/normal stress balances and the kinematic condition) become for $i=2,3$ (note that (2.18) is differentiated with respect to $\xi$ )

$$
\begin{gathered}
m_{(i-1)} \delta^{2} \tilde{w}_{(i-1) \xi}-m_{i} \delta^{2} \tilde{w}_{i \xi}+m_{(i-1)}\left(\bar{u}_{(i-1) y}+\tilde{u}_{(i-1) y}\right)-m_{i}\left(\bar{u}_{i y}+\tilde{u}_{i y}\right) \\
=-\frac{4 \delta^{2} h_{i \xi}}{1-\delta^{2} h_{i \xi}^{2}}\left(m_{(i-1)} \tilde{w}_{(i-1) y}-m_{i} \tilde{w}_{i y}\right) \\
\frac{\tilde{p}_{(i-1) \xi}-\tilde{p}_{i \xi}}{\delta}+\left(r_{i}-r_{(i-1)}\right) \cot \theta h_{i \xi}-\delta\left[\frac{1+\delta^{2} h_{i \xi}^{2}}{1-\delta^{2} h_{i \xi}^{2}}\left(m_{(i-1)} \tilde{w}_{(i-1) y}-m_{i} \tilde{w}_{i y}\right)\right]_{\xi} \\
=\left[\frac{\delta^{2} h_{i \xi \xi}}{C_{i}\left(1+\delta^{2} h_{i \xi}^{2}\right)^{3 / 2}}\right]_{\xi} \\
h_{i \tau}+\left(\bar{u}_{i}+\tilde{u}_{i}\right) h_{i \xi}-\tilde{w}_{i}=0 .
\end{gathered}
$$

In order to retain surface tension so that it competes with viscous stresses and density stratification forces in equation (2.30), we assume small capillary numbers and introduce the canonical limit $C_{i}=\delta^{2} \bar{C}_{i}$. The normal stress balance (2.30) becomes

$$
\begin{array}{r}
\frac{\tilde{p}_{(i-1) \xi}-\tilde{p}_{i \xi}}{\delta}+\left(r_{i}-r_{(i-1)}\right) \cot \theta h_{i \xi}-\delta\left[\frac{1+\delta^{2} h_{i \xi}^{2}}{1-\delta^{2} h_{i \xi}^{2}}\left(m_{(i-1)} \tilde{w}_{(i-1) y}-m_{i} \tilde{w}_{i y}\right)\right]_{\xi} \\
=\left[\frac{h_{i \xi \xi}}{\bar{C}_{i}\left(1+\delta^{2} h_{i \xi}^{2}\right)^{3 / 2}}\right]_{\xi}, \quad i=2,3 .
\end{array}
$$

Finally, the condition for the overall flow rate is given by

$$
\int_{0}^{h_{3}}\left(\bar{u}_{3}+\tilde{u}_{3}\right) d y+\int_{h_{3}}^{h_{2}}\left(\bar{u}_{2}+\tilde{u}_{2}\right) d y+\int_{h_{2}}^{1}\left(\bar{u}_{1}+\tilde{u}_{1}\right) d y=1 .
$$


We will construct asymptotic solutions of the problem that are periodic in $\xi$ of given scaled dimensionless period $2 L$, say; the quantity $L$ is a measure of the length of the system and is an important parameter in that it controls the number of linearly unstable modes about the trivial state.

\subsubsection{Long-wave coupled evolution equations}

Our objective is to derive a reduced-dimension system of equations that describes the nonlinear dynamics of the three-layer flow. The system originates from the dimensionless kinematic equations (2.31) evaluated at the appropriate asymptotic order, with the fluid mechanics in the bulk fixing the various terms by matching across interfaces. The following asymptotic expansions are introduced

$$
\left(\tilde{u}_{i}, \tilde{w}_{i}, \tilde{p}_{i}\right)=\left(\tilde{u}_{i}^{(0)}, \tilde{w}_{i}^{(0)}, \tilde{p}_{i}^{(0)}\right)+\delta\left(\tilde{u}_{i}^{(1)}, \tilde{w}_{i}^{(1)}, \tilde{p}_{i}^{(1)}\right)+\delta^{2}\left(\tilde{u}_{i}^{(2)}, \tilde{w}_{i}^{(2)}, \tilde{p}_{i}^{(2)}\right)+\ldots
$$

Substituting into (2.31) and retaining terms up to order $\delta$ we find

$$
h_{i \tau}+\bar{u}_{i} h_{i \xi}+\tilde{u}_{i}^{(0)} h_{i \xi}-\tilde{w}_{i}^{(0)}+\delta\left(\tilde{u}_{i}^{(1)} h_{i \xi}-\tilde{w}_{i}^{(1)}\right)=0 \quad \text { at } \quad y=h_{i}, \quad i=2,3 .
$$

This can be re-written in an integral form by using the continuity equation (2.28) and the no-slip boundary conditions (2.3) and (2.4), along with Liebniz's rule to obtain

$$
\begin{aligned}
& h_{2 \tau}+\left(\int \bar{u}_{1} d y+\int_{1}^{h_{2}} \tilde{u}_{1}^{(0)}+\delta \tilde{u}_{1}^{(1)} d y\right)_{\xi}=0 \text { at } y=h_{2}, \\
& h_{3 \tau}+\left(\int \bar{u}_{3} d y+\int_{0}^{h_{3}} \tilde{u}_{3}^{(0)}+\delta \tilde{u}_{3}^{(1)} d y\right)_{\xi}=0 \text { at } y=h_{3} .
\end{aligned}
$$

The leading order horizontal velocities $\tilde{u}_{i}^{(0)}$ are found by substituting (2.34) into the momentum equation (2.26) and retaining order one terms. The vertical momentum equation (2.27) gives $\tilde{p}_{i y}^{(0)}=0$, hence $\tilde{p}_{i}^{(0)} \equiv \tilde{p}_{i}^{(0)}(\xi, t)$, and the horizontal velocity profiles in each layer are parabolic in $y$ and given by

$$
\frac{m_{i}}{r_{i}} \tilde{u}_{i}^{(0)}=\frac{\tilde{p}_{i \xi}^{(0)}}{r_{i}} y^{2}+c_{4 i} y+c_{5 i}
$$

Here the nine functions $\tilde{p}_{i \xi}^{(0)}, c_{4 i}$ and $c_{5 i}, i=1,2,3$, depend on the physical parameters of the problem but also on the spatiotemporal interfacial dynamics $h_{i}(\xi, \tau), i=2,3$. They can be fully determined by considering the leading order contributions of the boundary conditions (2.3)-(2.5), (2.29), (2.32) and (2.33).

The horizontal momentum equation (2.26) at order $\delta$ allows us to solve for $\tilde{u}_{i}^{(1)}$ in terms of leading order quantities. The solution consists of two parts, one involving the pressure gradient and an inertial part, and is given by

$$
\frac{m_{i}}{r_{i}} \tilde{u}_{i}^{(1)}=\frac{\tilde{p}_{i \xi}^{(1)}}{r_{i}} y^{2}+R e \iint\left(\tilde{u}_{i \tau}^{(0)}+\tilde{w}_{i}^{(0)} \bar{u}_{i y}+\tilde{w}_{i}^{(0)} \tilde{u}_{i y}^{(0)}+\tilde{u}_{i \xi}^{(0)} \bar{u}_{i}+\tilde{u}_{i \xi}^{(0)} \tilde{u}_{i}^{(0)}\right) d y_{1} d y_{2}+c_{6 i} y+c_{7 i} .
$$

Equation (2.27) implies that $\tilde{p}_{i \xi}^{(1)}$ are also independent of $y$, but now also depend on the interfacial derivatives $h_{i \xi}$ and $h_{i \xi \xi \xi}$, as can be seen from the stress balance equation (2.32). The terms $\tilde{u}_{i \tau}^{(0)}$ can be calculated by differentiating equation (2.38) with respect to 
$\tau$ and using leading order terms of equations (2.36) and (2.37). Furthermore, the normal velocities $\tilde{w}_{i}^{(0)}$ can in turn be found from the continuity equation (2.28), to obtain

$$
\tilde{w}_{i}^{(0)}=-\int \tilde{u}_{i \xi}^{(0)} d y+c_{w i}, \quad i=1,2,3 .
$$

The functions $c_{w i}(\xi, \tau)$ can be found by applying the four boundary conditions (2.3)-(2.5) to the solution (2.40) and noting that the extra boundary condition (i.e. at $y=h_{2}$ ) is automatically satisfied due to the overall flow rate constraint (2.33) - see appendix A for details. Finally, substitution of (2.40) into (2.39) and use of the boundary conditions (2.3) -(2.5), (2.29) and (2.32) along with the condition of the fixed flow rate condition (2.33), enables the determination of the nine functions $\tilde{p}_{i \xi}^{(1)}, c_{6 i}$ and $c_{7 i}, \mathrm{i}=1,2,3$.

The leading order velocities appearing in equations (2.36) and (2.37) are now known and the final form of the long-wave coupled system that describes the spatiotemporal dynamics of the two interfaces takes the form

$$
h_{i \tau}+F_{i \xi}+\delta \sum_{j=1}^{2}\left[\left(R e S_{i j}+\cot \theta G_{i j}\right) h_{j \xi}+\frac{1}{\bar{C}_{i}} D_{i j} h_{j \xi \xi \xi}\right]_{\xi}=0, \quad i=2,3 .
$$

The $2 \times 1$ matrix $F_{i}$ and the $2 \times 2$ matrices $S_{i j}, G_{i j}, D_{i j}$ are rational polynomial functions of the interfacial deformations $h_{i}$ as well as the physical parameters of the problem $H_{i}$, $m_{i}$ and $r_{i}$. Their exact determination is tedious but straightforward and in this work we used the $M a t l a b^{\circledR}$ symbolic manipulation software for their efficient and error-free calculation. The system (2.41) is to be solved subject to periodic boundary conditions $h_{i}(\xi+2 L, t)=h_{i}(\xi, t)$.

Depending on the physical parameters, the leading order $(\delta=0)$ system (2.41) of conservation laws can be strictly hyperbolic in which case it would support shocks (thus violating the long-wave assumption), but can also be elliptic (or mixed hyperbolic-elliptic) making the system susceptible to short-wave instabilities and hence ill-posed. Consequently, the higher-order terms must be retained to regularize the equations in order to allow the possibility of long-time existence of the long-wave solutions. The reqularized equations (2.41) depend on the small parameter $\delta$ that cannot be scaled out of the problem. For a single interface (two fluid phases) the system reduces to a Benney-type equation (Bennev 1966), and it is well-known from the numerical work of Pumir et al. (1983) and Rosenau et al. (1992) that solutions can become unbounded in finite time for certain coefficients. Such difficulties motivate the derivation of canonical weakly nonlinear equations that do not contain $\delta$, directly from (2.41). Such equations provide the initial stages of the nonlinear dynamics by restricting the amplitudes to be small but not infinitesimal, and their derivation and study is considered next.

\section{Weakly nonlinear coupled evolution equations}

Here we aim to capture the initial nonlinear stages of the instability by imposing the restriction that the departure of the interfaces from the steady-state layer thicknesses is small (i.e. $\delta \eta_{i}=h_{(i+1)}-H_{(i+1)}$, where $\eta_{i}=O(1)$ ), and we can use Taylor expansions about the undisturbed states $H_{(i+1)}$. The system (2.41) now reduces to

$$
\eta_{i \tau}+\sum_{j=1}^{2}\left[q_{i j} \eta_{j \xi}+\sum_{k=1}^{2} \delta \beta_{i j k}\left(\eta_{j} \eta_{k}\right)_{\xi}+\delta\left(R e s_{i j}+\cot \theta g_{i j}\right) \eta_{j \xi \xi}+\frac{\delta}{\bar{C}_{i}} d_{i j} \eta_{j \xi \xi \xi \xi}\right]=0
$$


(a)

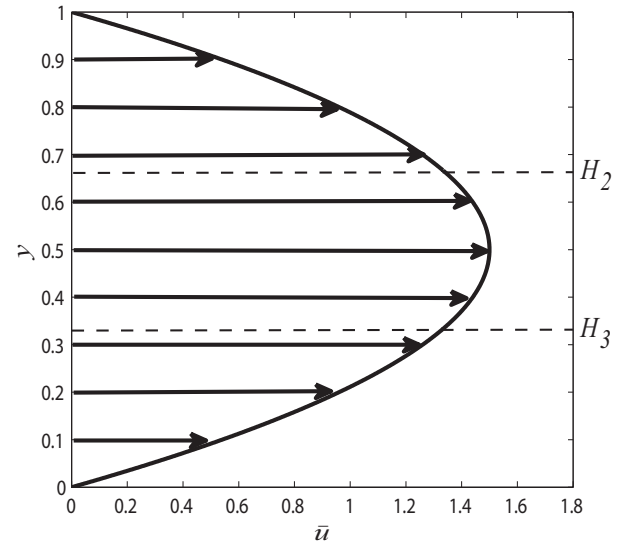

(b)

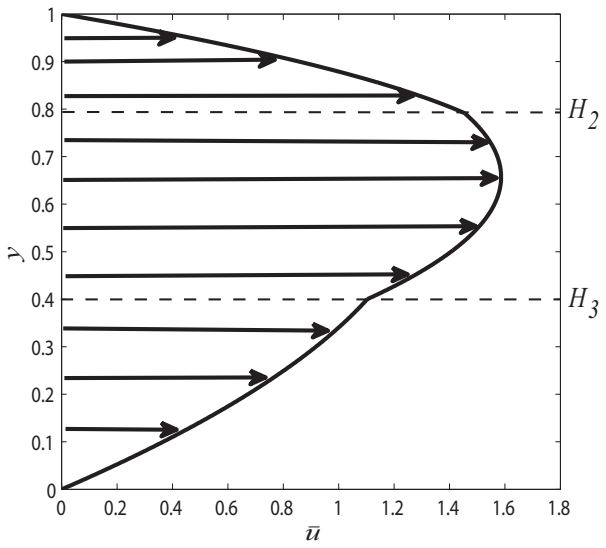

FIgURE 2. Steady-state velocity profiles for different fluid properties $\overline{\boldsymbol{T}}=\left(H_{2}, H_{3}, m_{2}, m_{3}, r_{2}, r_{3}\right)^{\top}$ : Panel $(a)$ symmetric case $\overline{\boldsymbol{T}}=\left(\frac{2}{3}, \frac{1}{3}, 1,1,1,1\right)^{\top} ;$ panel $(b)$ the non-symmetric case $\tilde{\mathbf{T}}=(0.79132,0.4,2,4.8457,1,1)^{\top}$.

where $i=1,2$. The constant coefficients $q_{i j}, \beta_{i j k}, s_{i j}, g_{i j}, d_{i j}$ are rational polynomial functions of the physical parameters of the system $\left(H_{i}, m_{i}, r_{i}\right)$. System (3.1) incorporates advective terms, Burgers-type coupled nonlinearities $\left(\eta_{j} \eta_{k}\right)_{\xi}$, buoyancy effect $\cot \theta \eta_{j \xi \xi}$, inertia $R e \eta_{j \xi \xi}$ and surface-tension $\frac{1}{C_{i}} \eta_{j \xi \xi \xi \xi}$ terms. The matrix of the surface tension is always positive definite providing damping for large wavenumbers; on the other hand, the matrix $g_{i j}$ provides damping for stably density stratified flows, and introduces RayleighTaylor instability otherwise. Furthermore, the matrix $g_{i j}$ vanishes when the densities of the three layers are equal.

We remark that a system of equations similar to (3.1) is valid for arbitrarily many interfaces constrained in an inclined channel. However, as can be observed the system (3.1) includes terms of different magnitude, since the advective term is of unity order while the other terms are of order $\delta$. As a result, the advective part of the system shadows the nonlinear dynamics. In order to retain the $\delta$-order dynamics, the advective term should be removed by an appropriate Galilean transformation as in the case of the single interface problem. However, in the case of two or more interfaces, this transformation is possible only if the matrix of the advective term is diagonal to leading order, with equal eigenvalues $\lambda$ say. Consequently, we proceed by defining the 6 -dimensional vector of the physical parameters of the problem $\boldsymbol{T}=\left(H_{2}, H_{3}, m_{2}, m_{3}, r_{2}, r_{3}\right)^{\top}$, where $0<H_{3}<H_{2}<1$, and identifying specific parameter vectors that allow a Galilean transformation. This analysis suggests two distinct cases corresponding to basic states that are symmetric or non-symmetric about $y=\frac{1}{2}$. We analyse these individually since they provide distinct dynamical systems.

\subsection{The symmetric case: Nonlinear advective-dissipative systems}

In this case the basic flow is parabolic and symmetric about $y=\frac{1}{2}$ as depicted in figure $2(a)$, and in general corresponds to the parameter vector $\boldsymbol{T} \equiv \overline{\boldsymbol{T}}=(1-\omega, \omega, 1,1,1,1)^{\top}$, where $0<\omega<\frac{1}{2}$. Importantly, this implies that the advective term is diagonal with equal eigenvalues $\lambda(\omega)$, so that $q_{i j}(\overline{\boldsymbol{T}})=\lambda(\omega) \delta_{i j}$, the non-linear terms $\beta_{i j k}(\overline{\boldsymbol{T}})=\bar{\beta}_{i}(\overline{\boldsymbol{T}})$ decouple, and the inertial and buoyancy terms become zero, i.e. $s_{i j}(\overline{\boldsymbol{T}})=0$ and $g_{i j}(\overline{\boldsymbol{T}})=$ 
$(a)$

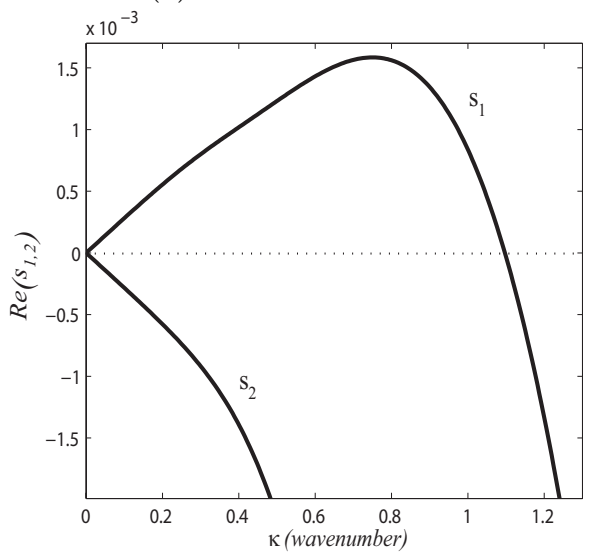

(b)

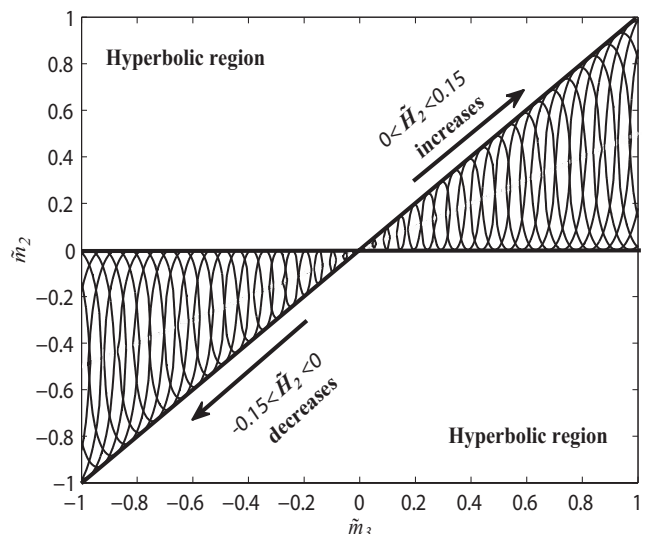

FIGURE 3. Linear stability results in the presence of the "alpha"-effect for the system (3.6) about the flat states $\overline{\boldsymbol{\eta}}=0$ when $\overline{\boldsymbol{T}}=\left(\frac{2}{3}, \frac{1}{3}, 1,1,1,1\right)^{\top}, \nu=1$ and $\frac{1}{C_{1}}=\frac{1}{C_{2}}=1$ : Panel $(a)$ growth rates with $\boldsymbol{Z}=\left(\frac{1}{10}, 0, \frac{78}{100}, \frac{8}{10}, 0,0\right)^{\top}$; panel $(b)$ marginal stability curves for $\boldsymbol{Z}=\left(\tilde{H}_{2}, 0, \tilde{m}_{2}, \tilde{m}_{3}, 0,0\right)^{\top}$.

0 . The resulting weakly nonlinear system (3.1) is

$$
\eta_{i \tau}+\lambda \eta_{i \xi}+\delta \bar{\beta}_{i}(\overline{\boldsymbol{T}})\left(\eta_{i}^{2}\right)_{\xi}+\delta \sum_{j=1}^{2} \frac{1}{\bar{C}_{i}} d_{i j}(\overline{\boldsymbol{T}}) \eta_{j \xi \xi \xi \xi}=0, \quad i=1,2 .
$$

It is easy to deduce that (3.2) is a stable advective-dissipative system (note that $d_{i j}$ is positive definite) with decoupled Burgers-type nonlinearities; consequently, at large times the system attains the trivial solution for rather general periodic initial conditions (e.g. a finite number of random Fourier coefficients) - this can be proven easily using simple energy estimates. In what follows we investigate these inertialess instabilities of the symmetric case by detuning the value of the parameter vector $\overline{\boldsymbol{T}}$ to derive general canonical systems.

We proceed by perturbing the system (3.1) about the symmetric case by writing $\boldsymbol{T}=$ $\overline{\boldsymbol{T}}+\delta \boldsymbol{Z}$, where $\boldsymbol{Z}=\left(\tilde{H}_{2}, \tilde{H}_{3}, \tilde{m}_{2}, \tilde{m}_{3}, \tilde{r}_{2}, \tilde{r}_{3}\right)^{\top}$ is a constant vector acting as a de-tuning parameter; retaining terms up to order $\delta$ yields

$$
\eta_{i \tau}+\lambda \eta_{i \xi}+\delta \bar{\beta}_{i}(\overline{\boldsymbol{T}})\left(\eta_{i}^{2}\right)_{\xi}+\delta \sum_{j=1}^{2}\left(q_{i j}^{(1)}(\overline{\boldsymbol{T}}, \boldsymbol{Z}) \eta_{j \xi}+\frac{1}{\bar{C}_{i}} d_{i j}(\overline{\boldsymbol{T}}) \eta_{j \xi \xi \xi \xi}\right)=0
$$

where

$$
q_{i j}^{(1)}(\overline{\boldsymbol{T}}, \boldsymbol{Z})=\sum_{l=1}^{6} Z_{l} \frac{\partial q_{i j}}{\partial T_{l}}(\overline{\boldsymbol{T}}), \quad i=1,2 .
$$

The vector $\bar{\beta}_{i}$ and matrix $d_{i j}$ depend on $\omega$ alone since they are leading order quantities. As an example, when $\overline{\boldsymbol{T}}=\left(\frac{2}{3}, \frac{1}{3}, 1,1,1,1\right)^{\top}$ (i.e. $\left.\omega=1 / 3\right)$, we have

$$
\bar{\beta}_{i}(\overline{\boldsymbol{T}})=\left(\begin{array}{c}
-1 \\
1
\end{array}\right), \quad d_{i j}(\overline{\boldsymbol{T}})=\left(\begin{array}{cc}
\frac{73}{100} & \frac{1}{2} \\
\frac{1}{2} & \frac{73}{100}
\end{array}\right),
$$

and emphasize that other physically relevant cases can be determined analogously.

To obtain the final form of the equations we perform a Galilean transformation $x^{*}=\xi-$ 
$(a)$

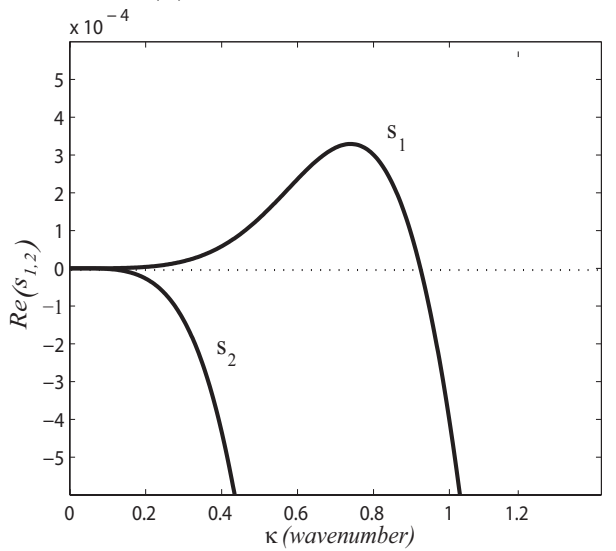

$(b)$

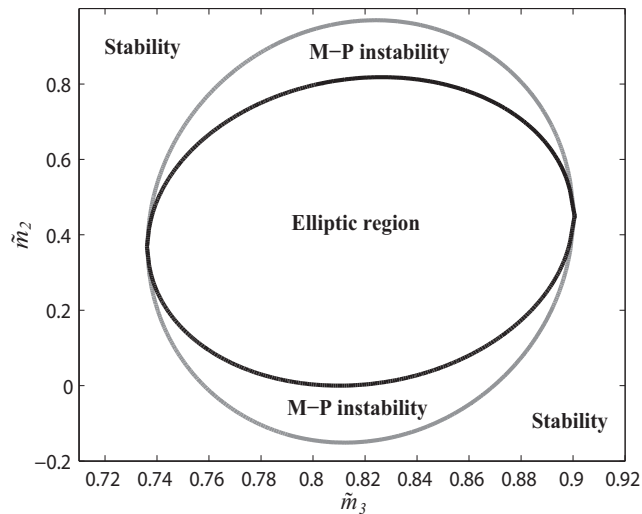

FIgURE 4. Linear stability results in the presence of the Majda-Pego instability for the system (3.6) about the flat states $\overline{\boldsymbol{\eta}}=0$ when $\overline{\boldsymbol{T}}=\left(\frac{2}{3}, \frac{1}{3}, 1,1,1,1\right)^{\top}, \nu=1$ and $\frac{1}{C_{1}}=\frac{1}{C_{2}}=1$ : Panel $(a)$ growth rates with $\boldsymbol{Z}=\left(\frac{93}{1000}, 0, \frac{78}{100}, \frac{8}{10}, 0,0\right)^{\top}$; panel $(b)$ marginal stability curves for $\boldsymbol{Z}=\left(\frac{93}{1000}, 0, \tilde{m}_{2}, \tilde{m}_{3}, 0,0\right)^{\top}$.

$\lambda \tau$, introduce a new slow time-scale $t^{*}=\delta \tau$, and normalize the equations to $2 \pi$-periodic domains by introducing new scaled coordinates $t^{* *}=\left(\frac{\pi}{L}\right)^{2} t^{*}, \quad x^{* *}=\left(\frac{\pi}{L}\right) x^{*}, \quad \eta_{i}^{*}=$ $\left(\frac{L}{\pi}\right) \eta_{i}$; the resulting bifurcation parameter $\nu=\left(\frac{\pi}{L}\right)^{2}>0$, where $2 L$ is the period of the solutions introduced earlier, plays a central role in the dynamics and is analogous to the "viscosity" parameter found in the Kuramoto-Sivashinsky equation. Dropping the asterisks yields the following system of nonlinear coupled evolution equations:

$$
\eta_{i t}+\bar{\beta}_{i}(\overline{\boldsymbol{T}})\left(\eta_{i}^{2}\right)_{x}+\sum_{j=1}^{2}\left(\frac{1}{\sqrt{\nu}} q_{i j}^{(1)}(\overline{\boldsymbol{T}}, \boldsymbol{Z}) \eta_{j x}+\frac{\nu}{\bar{C}_{i}} d_{i j}(\overline{\boldsymbol{T}}) \eta_{j x x x x}\right)=0, \quad i=1,2 .
$$

Previous work by Kliakhandler \& Sivashinsky 1995) derived model equations that are not of the canonical form derived here; the difference lies in the fact that the weakly nonlinear expansion was performed on a scaled Benney equation (equivalent to our equation (2.41) but with $\delta=1$ ), hence leading to a system with arbitrary coefficients in front of the advective and nonlinear terms. These terms play a crucial role in the instability mechanisms and nonlinear dynamics as we explain in more detail below and in Section 3. The canonical model (3.5) derived here, enables us to evaluate the competing physical effects of nonlinearity, advective instability due to the presence of two interfaces, and short-wave dissipation due to surface tension. An effective way to reveal the nature of the underlying instabilities is to rewrite the system in quasilinear matrix form as follows:

where

$$
\frac{\partial \boldsymbol{\eta}}{\partial t}+A(\boldsymbol{\eta}) \frac{\partial \boldsymbol{\eta}}{\partial x}+\nu D \frac{\partial^{4} \boldsymbol{\eta}}{\partial x^{4}}=0
$$

$$
\begin{gathered}
\boldsymbol{\eta}=\left(\begin{array}{c}
\eta_{1}(x, t) \\
\eta_{2}(x, t)
\end{array}\right), \quad A=\left(\begin{array}{cc}
-2 \eta_{1}(x, t)+\frac{q_{11}^{(1)}(\overline{\boldsymbol{T}}, \boldsymbol{Z})}{\sqrt{\nu}} & \frac{q_{12}^{(1)}(\overline{\boldsymbol{T}}, \boldsymbol{Z})}{\sqrt{\nu}} \\
\frac{q_{21}^{(1)}(\overline{\boldsymbol{T}}, \boldsymbol{Z})}{\sqrt{\nu}} & 2 \eta_{2}(x, t)+\frac{q_{22}^{(1)}(\overline{\boldsymbol{T}}, \boldsymbol{Z})}{\sqrt{\nu}}
\end{array}\right) \\
\text { and } \quad D \equiv \frac{1}{\bar{C}_{i}} d_{i j}(\overline{\boldsymbol{T}}) .
\end{gathered}
$$


The matrix $A$ varies in space and time and the nature of the instabilities depends crucially on its eigenvalues $\lambda_{1,2}(x, t)$. Given a solution $\boldsymbol{\eta}(x, t)$, these eigenvalues depend on $\boldsymbol{\eta}(x, t)$ and can be either real or complex conjugates. In the latter case the non-dissipative system becomes elliptic and induces catastrophic short-wave instabilities - i.e. the system is ill-posed with short waves growing the fastest (for a brief introduction to PDEs of mixed type the reader is referred to (Zauderer 2006); (Evans 2010)). Dissipation as it appears in equation (3.6) regularises such short-wave instabilities and more surprisingly can destabilise the long-wave modes when the eigenvalues are real. Noting that in our systems the diagonal elements of the dissipation matrix $d_{i j}$ are equal, then we find that such instabilities can only happen when the off-diagonal entries are non-zero. In what follows we illustrate these mechanisms with particular physical examples.

First we consider the linear stability of equation (3.6) about $\boldsymbol{\eta}=\overline{\boldsymbol{\eta}}=(0,0)^{\top}$ so that the eigenvalues of $A$ are complex. The flow is long-wave unstable to linear disturbances proportional to $e^{(i \kappa x+s t)}$, as depicted in figure 3( $(a)$. There are two modes with growth rates $s_{1}$ and $s_{2}$, the latter of which is stable for all wavenumbers $\kappa$. The effect of surface tension is to stabilise short waves and provide a finite band of instability, and the unstable mode has growth rates proportional to $\kappa$ for $\kappa<<1$. This instability is known as the "alpha"-effect - see (Kliakhandler \& Sivashinsky 1995) for references. We illustrate this by calculating the marginal stability of the system (3.6) for the case $\overline{\boldsymbol{T}}=\left(\frac{2}{3}, \frac{1}{3}, 1,1,1,1\right)^{\top}$, and $\boldsymbol{Z}=\left(\tilde{H}_{2}, 0, \tilde{m}_{2}, \tilde{m}_{3}, 0,0\right)^{\top}$. Physically, the chosen detuning parameter vector allows us to evaluate the role of viscosity and depth ratios. Figure $3(b)$ depicts the marginal stability curves; for each value of $\tilde{H}_{2}$ the marginal stability curve is a closed circular region in the $\tilde{m}_{2}-\tilde{m}_{3}$ plane. The figure shows a collection of these regions for $-\frac{15}{100} \leq \tilde{H}_{2} \leq \frac{15}{100}$, the locus of which defines the two triangular regions inside which the system is unstable. Furthermore, it can be observed that the instability vanishes when $\tilde{H}_{2}=0$, while it is present when the layer adjacent to the wall is more viscous than the intermediate one, i.e. $\tilde{m}_{2}<\tilde{m}_{3}$. The results also show that the instability region increases as $\tilde{H}_{2}$ increases, which corresponds to the upper layer depth decreasing with respect to the bottom layer depth. Analogous results hold for negative $\tilde{H}_{2}$, where now the upper layer depth increases relative to the bottom one and instability is found when $\tilde{m}_{2}>\tilde{m}_{3}$.

A more surprising and interesting finding is that even when $A$ possesses real and distinct eigenvalues, the system (3.6) can be unstable due to an interaction between the nonlinear flux and the dissipation terms due to surface tension. This kind of instability has been proposed in the context of viscously reqularized quasilinear systems by Majda \& Pego (1985). They studied strictly hyperbolic $2 \times 2$ systems of conservation laws with secondorder viscosity terms, i.e. $\boldsymbol{\eta}_{x x}$ in our notation, and show that when $A$ is symmetric and $D$ is positive definite, then their system (as well as ours) is stable for every constant state $\boldsymbol{\eta}=\overline{\boldsymbol{\eta}}$ satisfying

$$
\boldsymbol{l}_{i}(\overline{\boldsymbol{\eta}}) D \boldsymbol{r}_{i}(\overline{\boldsymbol{\eta}})>0
$$

where $\boldsymbol{l}_{i}$ and $\boldsymbol{r}_{i}, i=1,2$, are the left and right eigenvectors of $A$. One can also consider fixed viscosity matrices (in our notation the surface tension matrices $D$ ) and determine stable and unstable constant states (usually by studying Riemann problems - see (Canic \& Plohr 1995)). It follows, therefore, that solutions $\boldsymbol{\eta}$ of (3.6) can be unstable in the sense of Majda and Pego for fixed values of $x$ and $t$ when (note that we rewrite (3.7) - see proposition (5.12) in (Canic \& Plohr 1995))

$$
\frac{\operatorname{tr}\left(D^{-1}\left[-\lambda_{i}(x, t) \boldsymbol{I}+\boldsymbol{A}(\boldsymbol{\eta})\right]\right)}{\operatorname{tr}\left(-\lambda_{i}(x, t) \boldsymbol{I}+\boldsymbol{A}(\boldsymbol{\eta})\right)}<0,
$$


where $I$ is the identity matrix, for either $i=1$ or 2 . The Majda-Pego instability in our problem is illustrated next by the linear stability about $\boldsymbol{\eta}=\overline{\boldsymbol{\eta}}=(0,0)^{\top}$, for slightly different flux function and dissipation matrix as in figure 3 , and slightly different values of $\boldsymbol{Z}$ to place us just outside the elliptic region. Typical results are depicted in figure $4(a)$ with parameter values shown in the caption; the results show that in the long-wave limit $\kappa<<1$, we have $\operatorname{Re}(s) \sim \kappa^{2}$, indicating the different nature of the dissipative mechanism of the instability compared to the kinematic one discussed earlier. The boundary of the region inside which Majda-Pego instability occurs is depicted in figure $4(b)$ by the outer gray closed circular curve which is constructed using the condition (3.8). The topology of the elliptic, Majda-Pego and hyperbolic regions shown in figure 4( $b)$ is quite generic and has been described by Peters \& Canic (2000) in the context of three-phase reservoir flow in porous media. Note that condition (3.8) will be used in our numerical computations to determine the presence or absence of Majda-Pego instability of nonlinear solutions in the spatiotemporally evolving dynamics. This condition is clearly much stronger than the linear conditions about the null state used by Kliakhandler \& Sivashinsky (1995). We remark that the analysis of Majda \& Pego (1985) applies to second-order viscosity matrices; to our knowledge the analysis for higher order dissipation, e.g. fourth-order as in the present problem, remains open. Our numerical results indicate that condition (3.8) is still valid for our dissipation matrices in (3.6).

\subsection{The non-symmetric case: Kinematically modified coupled Kuramoto-Sivashinsky systems}

In this case the overall steady-state velocity profiles do not possess any symmetry about the channel midplane as depicted in figure $2(b)$. This case corresponds, in general, to the following parameter vector $\boldsymbol{T} \equiv \tilde{\boldsymbol{T}}=\left(H_{2}, H_{3}, m_{2} \neq 1, m_{3} \neq 1, r_{2} \neq 1, r_{3} \neq 1\right)^{\top}$. Hence, the system (3.1) can be written as follows:

$$
\begin{aligned}
\eta_{i \tau}+\sum_{j=1}^{2}\left[q_{i j}(\tilde{\boldsymbol{T}}) \eta_{i \xi}+\right. & \sum_{k=1}^{2} \delta \beta_{i j k}(\tilde{\boldsymbol{T}})\left(\eta_{j} \eta_{k}\right)_{\xi}+\delta\left(\operatorname{Res} s_{i j}(\tilde{\boldsymbol{T}})+\cot \theta g_{i j}(\tilde{\boldsymbol{T}})\right) \eta_{j \xi \xi} \\
& \left.+\frac{\delta}{\bar{C}_{i}} d_{i j}(\tilde{\boldsymbol{T}}) \eta_{j \xi \xi \xi \xi}\right]=0, \quad i=1,2 .
\end{aligned}
$$

As previously the advective term dominates the order $\delta$ dynamics. Hence, we can remove it by a Gallilean transformation by identifying parameter vectors $\tilde{\boldsymbol{T}}=\tilde{\boldsymbol{T}}_{\boldsymbol{d}}$ that make the matrix $q_{i j}\left(\tilde{\boldsymbol{T}}_{d}\right)$ diagonal with equal eigenvalues $\lambda$, yielding, correct to order $\delta$ :

$$
\begin{gathered}
\eta_{i \tau}+\sum_{j=1}^{2}\left[\lambda \eta_{i \xi}+\sum_{k=1}^{2} \delta \beta_{i j k}\left(\tilde{\boldsymbol{T}}_{\boldsymbol{d}}\right)\left(\eta_{j} \eta_{k}\right)_{\xi}+\delta\left(R e s_{i j}\left(\tilde{\boldsymbol{T}}_{\boldsymbol{d}}\right)+\cot \theta g_{i j}\left(\tilde{\boldsymbol{T}}_{\boldsymbol{d}}\right)\right) \eta_{j \xi \xi}\right. \\
\left.+\frac{\delta}{\bar{C}_{i}} d_{i j}\left(\tilde{\boldsymbol{T}}_{\boldsymbol{d}}\right) \eta_{j \xi \xi \xi \xi}\right]=0, \quad i=1,2 .
\end{gathered}
$$

Note that the buoyancy term $g_{i j}$ vanishes in the case of equal densities, and we proceed with this term absent - similar systems that have analogous qualitative behaviour can be derived when the densities are unequal. Physically, the presence of Rayleigh-Taylor instability (more dense fluid on top of lighter fluid), will produce linear instabilities with the combined matrix $\operatorname{Re} s_{i j}\left(\tilde{\boldsymbol{T}}_{\boldsymbol{d}}\right)+\cot \theta g_{i j}\left(\tilde{\boldsymbol{T}}_{\boldsymbol{d}}\right)$ being positive definite. The system (3.10), in contrast to (3.2), involves coupled nonlinearities as well as inertial terms (of order $\delta$ ). An asymptotically correct canonical system that also retains kinematic terms 
follows by introducing $\tilde{\boldsymbol{T}}=\tilde{\boldsymbol{T}}_{\boldsymbol{d}}+\delta \boldsymbol{Z}$ into (3.10), and performing an appropriate Galilean transformation to find

$$
\begin{gathered}
\eta_{i t}+\sum_{j=1}^{2}\left[\frac{1}{\sqrt{\nu}} q_{i j}^{(1)}\left(\tilde{\boldsymbol{T}}_{\boldsymbol{d}}, \boldsymbol{Z}\right) \eta_{j x}+\sum_{k=1}^{2} \beta_{i j k}\left(\tilde{\boldsymbol{T}}_{\boldsymbol{d}}\right)\left(\eta_{j} \eta_{k}\right)_{x}+\operatorname{Re} s_{i j}\left(\tilde{\boldsymbol{T}}_{\boldsymbol{d}}\right) \eta_{j x x}\right. \\
\left.+\frac{\nu}{\bar{C}_{i}} d_{i j}\left(\tilde{\boldsymbol{T}}_{\boldsymbol{d}}\right) \eta_{j x x x x}\right]=0, \quad i=1,2,
\end{gathered}
$$

where $q_{i j}^{(1)}\left(\tilde{\boldsymbol{T}}_{\boldsymbol{d}}, \boldsymbol{Z}\right)$ is defined by (3.4). In this study we introduce a constant parameter $\zeta$ so that $\mathbf{Z} \rightarrow \zeta \mathbf{Z}$ to enable us to control the size of the advective term relative to inertia (as we will see later, the competition between these terms influences the large time dynamics and resulting coherent structures). However, retaining the six independent parameters $Z_{l}, l=1, \ldots, 6$ provides the freedom to control the numerical values of $q_{i j}$. Equations (3.11) are a kinematically modified coupled Kuramoto-Sivashinsky (cKS) system and contains all the physical mechanisms of the single KS equation in addition to the instability mechanisms described in Section 3.1, Our main objective is to describe the ensuing nonlinear dynamics of such complex systems. We proceed by rewriting (3.11) in the following matrix form

$$
\frac{\partial \boldsymbol{\eta}}{\partial t}+\frac{\partial Q(\boldsymbol{\eta} ; \zeta, \nu)}{\partial x}+S \frac{\partial^{2} \boldsymbol{\eta}}{\partial x^{2}}+\nu D \frac{\partial^{4} \boldsymbol{\eta}}{\partial x^{4}}=0,
$$

where the matrix $S \equiv s_{i j}$ is positive definite and the flux function $Q(\boldsymbol{\eta})$ is the following non-homogeneous quadratic polynomial

$$
Q=\left(\begin{array}{c}
\beta_{111} \eta_{1}^{2}+\left(\beta_{112}+\beta_{121}\right) \eta_{1} \eta_{2}+\beta_{122} \eta_{2}^{2}+\frac{\zeta}{\sqrt{\nu}} q_{11}^{(1)} \eta_{1}+\frac{\zeta}{\sqrt{\nu}} q_{12}^{(1)} \eta_{2} \\
\beta_{211} \eta_{1}^{2}+\left(\beta_{212}+\beta_{221}\right) \eta_{1} \eta_{2}+\beta_{222} \eta_{2}^{2}+\frac{\zeta}{\sqrt{\nu}} q_{21}^{(1)} \eta_{1}+\frac{\zeta}{\sqrt{\nu}} q_{22}^{(1)} \eta_{2}
\end{array}\right)
$$

We denote the eigenvalues of the Jacobian $\nabla_{\eta} Q$ by $\lambda_{1,2}(x, t)$. Note that the Reynolds number $R e$ has been removed from the problem by rescaling $\eta_{1,2}$ and time $t$. The problem has two important parameters measuring competing mechanisms: $\zeta$ corresponding to kinematic effects and $\nu$ measuring the size of the system. In what follows we solve the problem numerically in order to describe the effect of these parameters on the nonlinear dynamics.

\section{Numerical experiments}

We begin by describing the numerical schemes used to solve the canonical systems (3.6) and (3.12). The spatial domains are $2 \pi-$ periodic and random initial conditions are used unless otherwise stated. We use spectral methods for the spatial discretisations with implicit time-stepping due to the stiffness of the fourth order derivatives (we implemented and tested the $M a t l a b{ }^{\circledR}$ integrator ode23tb as well as home-grown explicit-implicit BDF algorithms - (Akrivis et al. 2009)). Briefly, we represent the solutions by their Fourier series

$$
\eta_{i}(x, t)=\sum_{\mu=1}^{\infty}\left(\eta_{i \mu}^{c}(t) \cos \mu x+\eta_{i \mu}^{s}(t) \sin \mu x\right),
$$

to find an infinite-dimensional system of coupled nonlinear ODEs for the Fourier coefficients $\eta_{i \mu}^{c}(t), \eta_{i \mu}^{s}(t)$. The main difficulty involves the Fourier representation of the nonlinerities - this is done analytically and can be found in (Akrivis et al. 2009), for instance. The system is truncated to $M$ terms (we are assuming that the dynamics are low-modal 
as in the case of the single KS equation, and this is checked numerically a posteriori) and solved as an initial value problem using a stiff ODE solver to maintain stability and accuracy; the analytical expressions for the nonlinearities are computed efficiently (i.e. with an operation count which is much less than $O\left(M^{2}\right)$ ) using vectorised matrix multiplications provided by Matlab $^{\circledR}$.

One of the diagnostics we use in order to check boundedness of solutions is the evolution of their energy (or $L_{2}$-norm). Parseval's theorem shows that the $L_{2}$-norm can be calculated spectrally and is given by the following expression:

$$
E_{i}(t) \equiv\left\|\eta_{i}\right\|_{2}=\sqrt{\pi \sum_{\mu=1}^{M}\left(\left(\eta_{i \mu}^{c}\right)^{2}+\left(\eta_{i \mu}^{s}\right)^{2}\right)}, \quad i=1,2 .
$$

Furthermore, in order to construct the phase plane $\left(E_{i}, \dot{E}_{i}\right)$ of the solutions we differentiate (4.2) with respect to $t$ to obtain

$$
\dot{E}_{i}=\frac{\sqrt{\pi} \sum_{\mu=1}^{M}\left(2 \eta_{i \mu}^{c} \frac{d \eta_{i \mu}^{c}}{d t}+2 \eta_{i \mu}^{s} \frac{d \eta_{i \mu}^{s}}{d t}\right)}{2\left\|\eta_{i}\right\|_{2}}, \quad i=1,2 .
$$

Expression (4.3) is spectrally accurate making the phase plane characteristics spectrally accurate also. This is important in determining complex dynamics (e.g. period-doubling bifurcation routes to chaos) from the numerical data. Our numerical diagnostic tools have been described elsewhere - see (Akrivis et al. 2012), (Smyrlis \& Papageorgiou 1991). In what follows we describe results for the symmetric and non-symmetric cases, respectively, i.e. equations (3.6) and (3.12).

\subsection{Interfacial dynamics - Nonlinear advective-dissipative systems}

In this section, we investigate numerically the interfacial dynamics exhibited by the advection-dissipative system (3.6). The initial conditions typically contain the first ten harmonics with amplitudes chosen randomly in the interval $[-0.5,0.5]$. The number of modes $M$ depends on the parameter $\nu$ and $M$ is always chosen so that the numerical solution has an exponentially decaying spectrum in Fourier space.

The large time behaviour of the solutions depends crucially on the eigenvalues of matrix $A$ in (3.6). Denoting these by $\lambda_{1,2}$ we find that they are real or complex conjugates depending on whether the sign of $\mathcal{D}$, defined by,

$$
\mathcal{D}=\frac{1}{\nu}\left[\left(-2 \sqrt{\nu}\left(\eta_{1}(x, t)+\eta_{2}(x, t)\right)+q_{11}-q_{22}\right)^{2}+4 q_{12} q_{21}\right],
$$

is positive or negative, respectively. In the former case the flux function is hyperbolic and as a result (due to the presence of diffusion) the solutions decay to zero at large times independent of initial conditions - a sufficient condition for this to happen is $q_{12} q_{21} \geq 0$. Hence, we concentrate on situations where the flux function can provide mixed type (i.e. hyperbolic-elliptic) behaviour.

The eigenvalues $\lambda_{1,2}$ depend on the values of $q_{i j}$ (recall that these correspond to different physical situations and can be chosen to take a wide range of values), and the initial conditions. For example, if the $q_{i j}$ are such that the flat state is linearly unstable (either due to Majda-Pego instability or the "alpha" effect), then any arbitrary initial condition will evolve to nonlinear travelling wave states. We illustrate this scenario in figure 5 for 
$\nu=1$ (other parameters given in the caption) and $q_{i j}$ given by

$$
q_{i j}=\left(\begin{array}{cc}
-0.519 & 0.123 \\
-0.247 & -0.341
\end{array}\right) .
$$

The figure shows the evolution of $\eta_{1}(x, t)$ and $\eta_{2}(x, t)$ in panels $(a)$ and $(b)$ correspondingly, and the corresponding evolution of their energy norms (panel $(c)$ ), indicating that the solutions evolve to finite energy travelling wave states. These phenomena can be understood by considering the spatiotemporal evolution of the matrix $A$ and in particular the nature of its eigenvalues according to (4.4). As the solutions $\eta_{1,2}(x, t)$ evolve, we track the regions in the $x-t$ plane where $\mathcal{D}$ takes on negative (black) or positive (gray) values as shown in panel $(d)$. In addition we indicate regions of Majda-Pego instability (see (3.8)) with a white colour and these can be found on the edges of the elliptic regions (as described earlier also - see figure (4). Even though the systems studied here are parabolic (fourth order diffusion), non-trivial states can only emerge if there exist dynamic transitions resulting from the nonlinearities and their interaction with the damping; these transitions (hyperbolic to elliptic) are the hallmark of the emerging dynamics.

Through extensive numerical experiments we have established that when the flat states are linearly stable, the emerging dynamics depend on the energy input of the initial conditions. If the initial energy is below a threshold value (this value clearly depends on problem parameters, e.g. $\nu$ ) then a trivial state emerges at large time, whereas above threshold non-trivial travelling wave states emerge as found earlier. Results near threshold for the case $\nu=1$ and

$$
q_{i j}=\left(\begin{array}{cc}
-1 & 0.123 \\
-0.247 & -0.341
\end{array}\right)
$$

are given in figure 6 (other parameters given in the caption). The upper panels $(a),(b)$ are below threshold (the initial energy input is 1.1636), while the lower ones $(c),(d)$ are just above (with energy 1.1639). The right figures depict the type of the flux function eigenvalues as explained earlier. In both cases (below and above threshold) there exist elliptic regions initially, and these either disappear or persist depending on the initial energy. Below threshold, the damping is sufficient to drive the system to a uniformly hyperbolic state thus leading to trivial solutions, whereas above threshold ellipticity persists leading to non-trivial states. We can conclude, therefore, that the linear stability is a necessary but not sufficient condition for the emergence of non-trivial nonlinear states.

\subsection{Interfacial dynamics - Kinematically modified coupled Kuramoto-Sivashinsky systems}

In this Section we study flows that include inertia and are governed by the coupled Kuramoto-Sivashinsky systems (3.12). We carried out extensive numerical experiments as the parameters $\nu$ and $\zeta$ vary. In the results that follow we take the vector of the physical parameters to be $\tilde{\mathbf{T}}=(0.79132,0.4,2,4.8457,1,1)^{\top}$ and the detuning vector $\mathbf{Z}=(0.1,0,8,8,0,0)^{\top}$. These values correspond to a physical situation with the upper and lower undisturbed interfaces at $H_{2}=0.79132, H_{3}=0.4$, and the viscosity ratios $m_{2}=2, m_{3}=4.8457$ (in this scenario the upper layer is less viscous than the other layers and the lower layer is the most viscous); the densities of the three layers are equal in this example. With these values the matrices $\beta_{1 j k}, \beta_{2 j k}, s_{i j}, d_{i j}, q_{i j}$ are calculated to be

$$
\beta_{1 j k}=\left(\begin{array}{cc}
-1.5774 & -0.0209 \\
-0.0209 & 0.1919
\end{array}\right), \quad \beta_{2 j k}=\left(\begin{array}{cc}
0.2477 & -0.0509 \\
-0.0509 & 1.3278
\end{array}\right),
$$


$(a)$

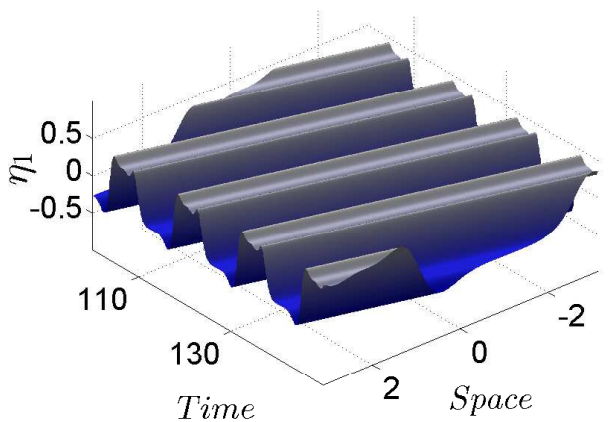

(c)

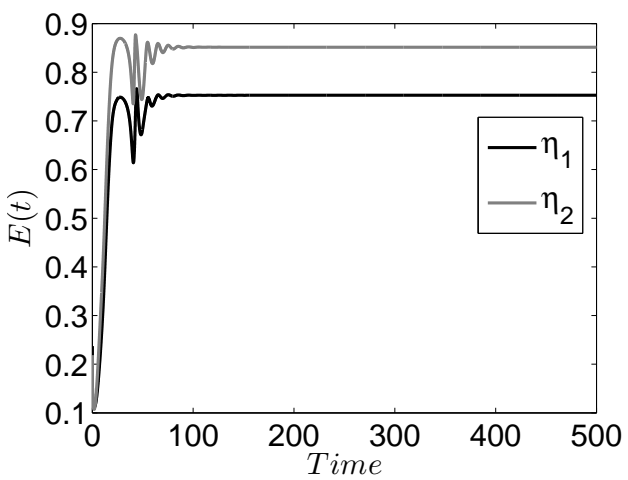

$(b)$

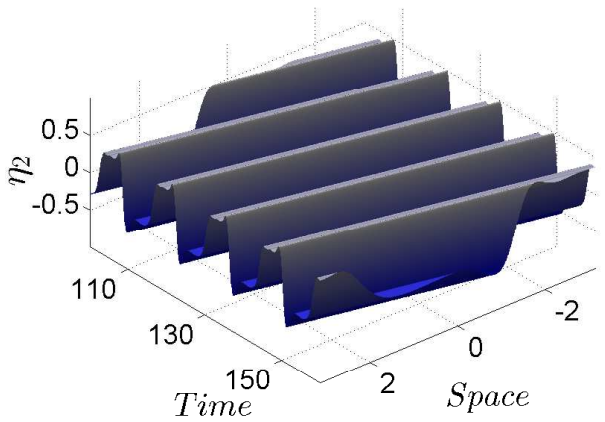

(d)

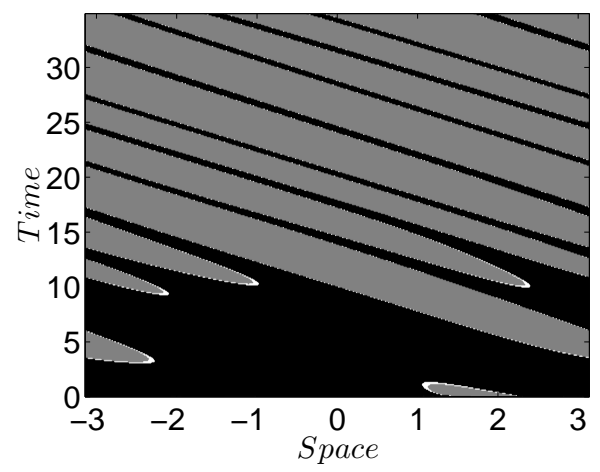

Figure 5. Emergence of steady-state traveling waves of system (3.6), when $\overline{\boldsymbol{T}}=\left(\frac{2}{3}, \frac{1}{3}, 1,1,1,1\right)^{\top}, \boldsymbol{Z}=(1,0.9405,10,15,0,0)^{\top}, \nu=1$ and $\frac{1}{C_{1}}=\frac{1}{C_{2}}=1$. The matrix of the advective term $q_{i j}$ possesses conjugate complex eigenvalues. Panels $(a)$ and $(b)$, the spatiotemporal evolution of the interfaces; panel $(c)$, the evolution of the energy; panel $(d)$, the spatiotemporal evolution of underlying instability regions: where black-shaded regions correspond to complex eigenvalues, white-shaded regions to Majda-Pego instability and gray-shaded regions indicate hyperbolicity.

$$
\begin{gathered}
s_{i j}=\left(\begin{array}{cc}
-5.30615 & 5.4011 \\
-5.2902 & 8.2255
\end{array}\right) \times 10^{-4}, \quad d_{i j}=\left(\begin{array}{cc}
0.0020 & 0.0015 \\
0.0015 & 0.0033
\end{array}\right), \\
q_{i j}=\left(\begin{array}{cc}
-0.445 & 0.052 \\
-0.075 & -0.504
\end{array}\right) .
\end{gathered}
$$

Note also that with this choice of $\tilde{\mathbf{T}}$ we ensure that the nonlinear flux function is of mixed hyperbolic-elliptic type, thus avoiding strictly hyperbolic nonlinearities that could provide more standard Kuramoto-Sivashinsky dynamics. In addition, the detuning parameter $\zeta$ is selected to provide kinematic instability of the zero states, i.e. the matrix $q_{i j}$ has complex eigenvalues. This example may be difficult to access experimentally due to the simultaneous variation of four physical parameters in the detuning vector. Nevertheless, analogous dynamics have been found for simpler cases that vary the layer depths alone (e.g. $\boldsymbol{Z}=(-0.0799,0.1,0,0,0,0)^{\top}$ that increases the thicknesses of the undisturbed upper and lower layers). 
(a)

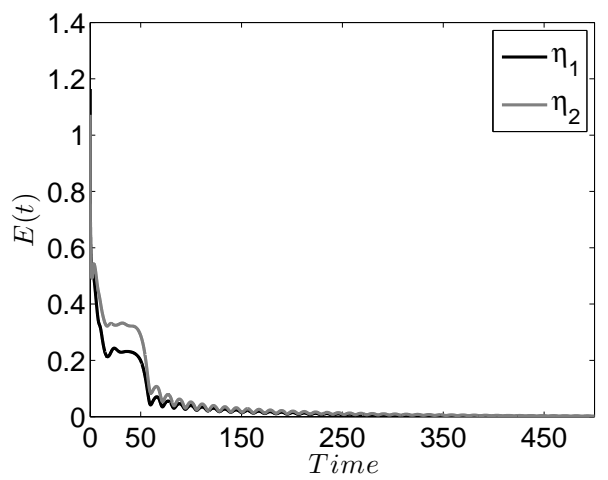

(c)

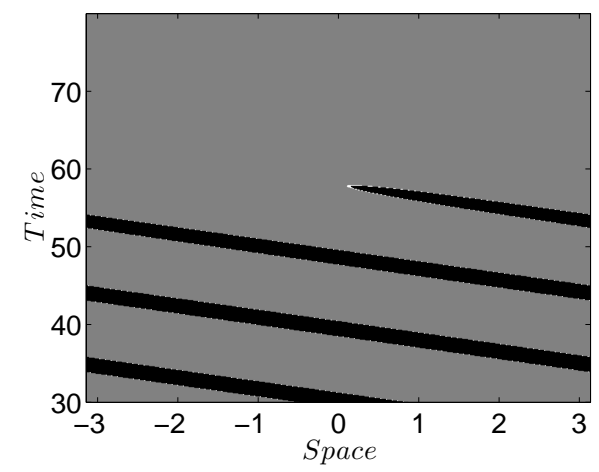

(b)

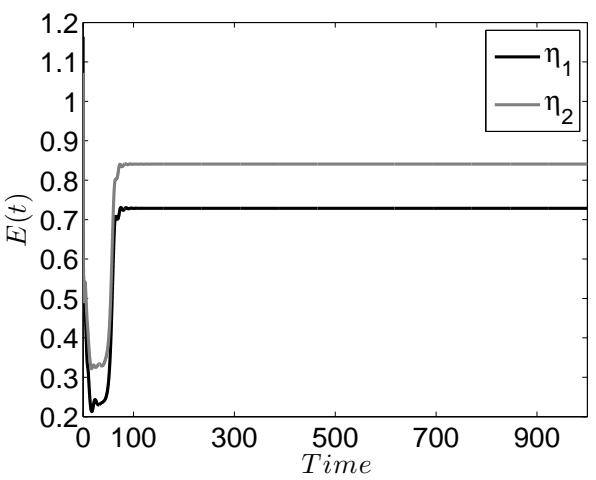

(d)

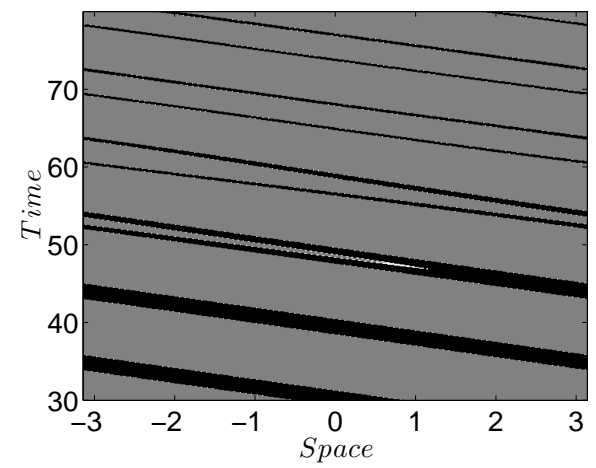

FIGURE 6. Dependence of long-time behavior on initial energy input for the the linearly stable system (3.6), with $\overline{\boldsymbol{T}}=\left(\frac{2}{3}, \frac{1}{3}, 1,1,1,1\right)^{\top}, \boldsymbol{Z}=(1.2405,0.9405,10,15,0,0)^{\top}, \nu=1$ and $\frac{1}{C_{1}}=\frac{1}{C_{2}}=1$. Panels $(a)$ and $(b)$ have initial conditions below threshold, while panels $(c)$ and $(d)$ correspond to conditions above threshold.The energy evolution is shown along with the evolution of the instability regions (black-shaded regions correspond to complex eigenvalues, white-shaded regions to Majda-Pego instability and gray-shaded regions indicate hyperbolicity).

Our main interest here is in quantifying the kinematic effect through the parameter $\zeta$. We present numerical results as $\zeta$ varies for a fixed value of $\nu=0.003$ to ensure that we start with chaotic dynamics when $\zeta=0$ as seen in figure 7(a),(b). Panel (a) shows the evolution of the energy norms of $\eta_{1}$ and $\eta_{2}$ and panel (b) shows the corresponding phase plane of $\eta_{1}$. We note that evidence of chaotic dynamics is deduced from the everincreasing and non-overlapping number of loops in the phase plane as time increases (this was quantified further by constructing return maps (see Akrivis et al. 2012) - but these are not included for brevity). Note that in this case both inertial and inertialess instabilities contribute to the dynamics of the system. Cases where the energy input due to inertia dominates are considered later and are interesting due to the fact that the dynamics are closer to those of the single Kuramoto-Sivashinsky equation. The effect of increasing $\zeta$ is to regularise the dynamics from chaos to time-periodic travelling waves and ultimately to steady-state nonlinear travelling waves. Table 1 contains the first three windows as $\zeta$ increases that show this regularisation. We note that for $\zeta$ larger than approximately $0.44184 \times 10^{-3}$, the dynamics is of type I, II or III (as categorised in the Table), but when $\zeta$ is larger than 0.0026 , approximately, the flow is attracted to steady- 
(a)

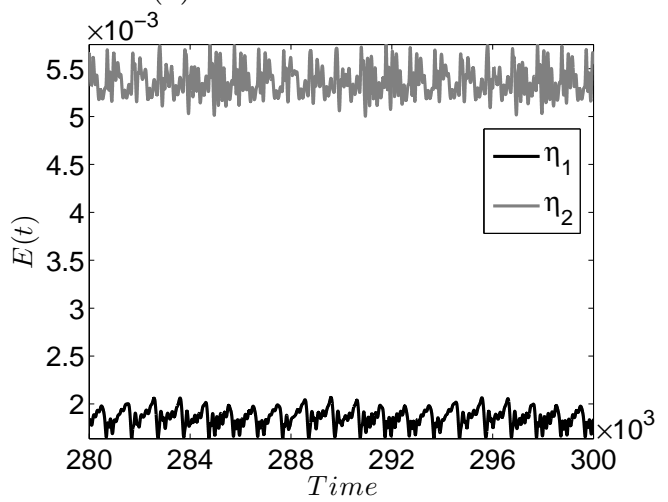

(c)

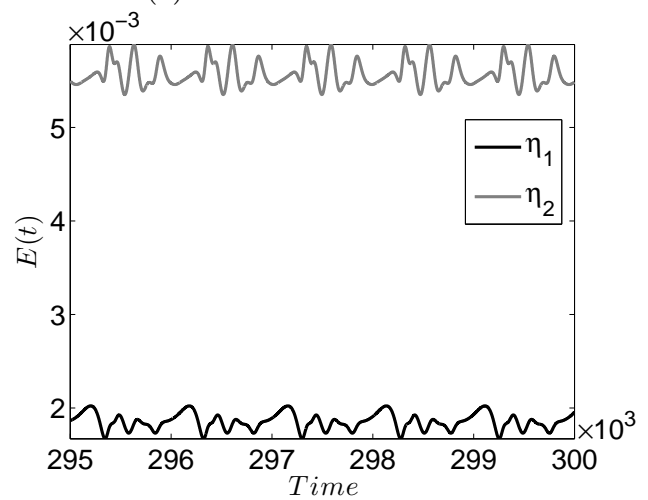

(b)

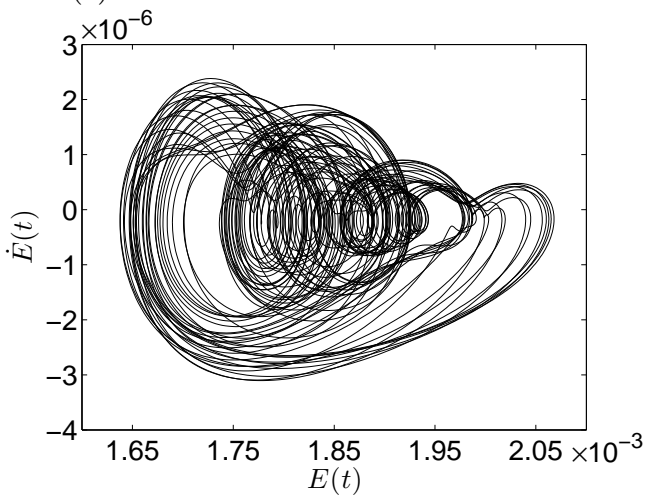

$(d)$

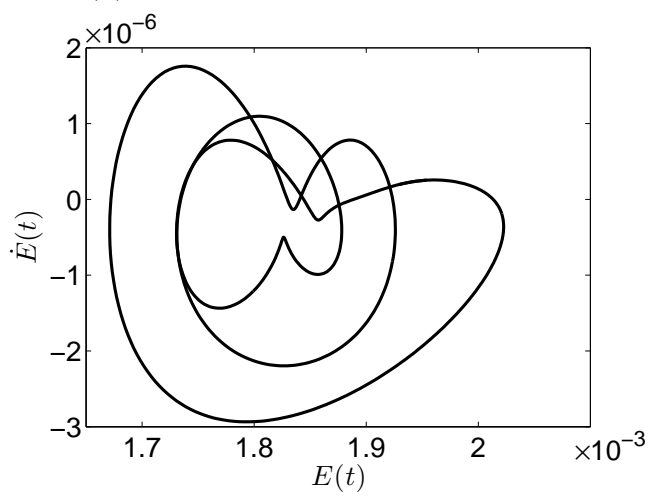

Figure 7. Regularization of chaotic oscillations for the non-symmetric system (3.12) as the value of the parameter $\zeta$ increases for fixed $\nu=0.003$ and $\frac{1}{C_{1}}=\frac{1}{C_{2}}=1$. Panels $(a)$ and $(b)$ have $\zeta=0$ and give chaotic dynamics, while panels $(c)$ and $(d)$ correspond to $\zeta=0.15811 \times 10^{-3}$ and provide time-periodic travelling waves.The energy evolution is shown along with the phase planes of $\left(E_{1}(t), \dot{E}_{1}(t)\right)$.

state nonlinear travelling waves. Figure $7(\mathrm{c}),(\mathrm{d})$ shows a typical time-periodic travelling wave from attractor II having $\zeta=0.15811 \times 10^{-3}$. The energy norms (panel (c)) are time periodic signals and this is clearly seen in the phase plane in panel (d) that is seen to contain three repeating loops.

In what follows we solve an analogous system as the one above with the same matrices $\beta_{1 j k}, \beta_{2 j k}, d_{i j}, q_{i j}$ but with a different inertia-associated matrix given by

$$
s_{i j}=\left(\begin{array}{cc}
3.5655 & 3.4635 \\
-4.0635 & -7.6411
\end{array}\right) \times 10^{-4} .
$$

This model is motivated by the fact that the energy input due to the inertia-associated matrix $s_{i j}$ given by (4.5) dominates and produces slaved dynamics, meaning that the dynamics of one interface lock into those of the other, resulting in mostly hyperbolic nonlinearities. Hence the inertia terms due to (4.5) lead to mostly single KS-type dynamics when $\zeta=0$. Next we quantify the effect of $\zeta$ with particular interest in the way that it affects the slaved dynamics mentioned above - we illustrate this by starting with the case $\nu=0.015$ and $\zeta=0$. Results are given in figure 8 that depicts the energy norms of $\eta_{1}(x, t)$ and $\eta_{2}(x, t)$. The large time dynamics are chaotic homoclinic bursts (the solution is attracted to steady states which then loose stability, become chaotic and then 


$\begin{array}{ccc}\text { Attractor } & \zeta & \text { Description } \\ I & \lesssim 0.82056 \times 10^{-4} & \text { chaotic oscillations } \\ \text { II } & \lesssim 0.28088 \times 10^{-3} & \text { periodic travelling waves } \\ \text { III } & \lesssim 0.44184 \times 10^{-3} & \text { steady-state travelling waves }\end{array}$

TABLE 1. Attractors of the kinematically modified cKS system for fixed $\nu=0.003$ and $\frac{1}{C_{1}}=\frac{1}{C_{2}}=1$; The values of $\zeta$ denote an approximate upper bound for the described attractor.

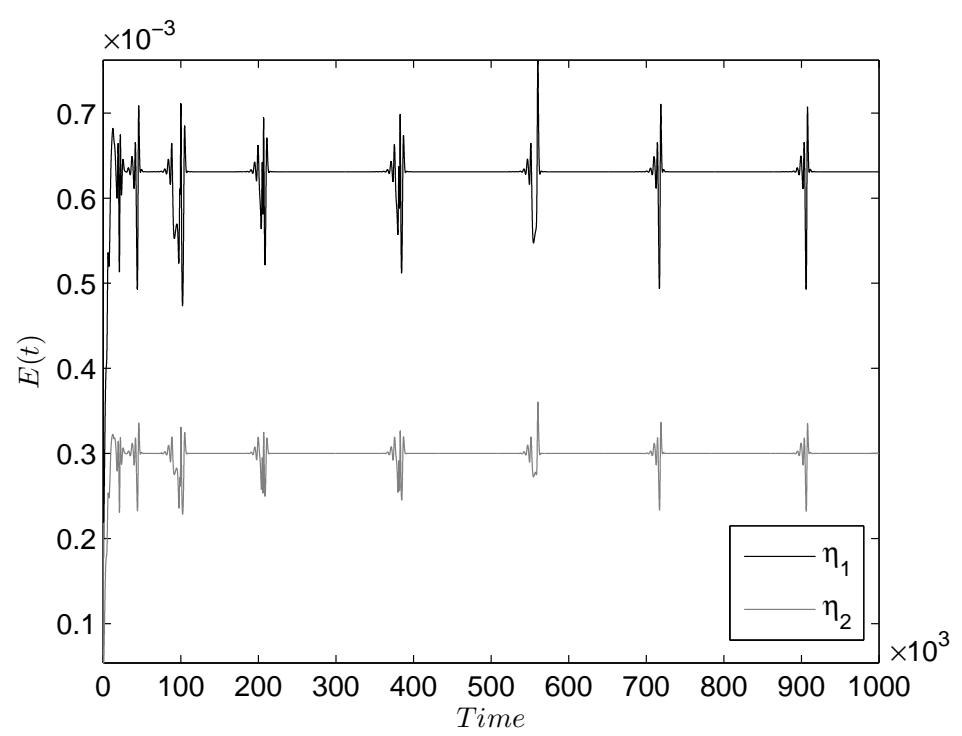

FIGURE 8. The evolution of the energy norms for the non-symmetric system (3.12) when the inertia-associated matrix $s_{i j}$ is given by (4.5). Emergence of chaotic homoclinic bursts for $\zeta=0$, $\nu=0.015$ and $\frac{1}{C_{1}}=\frac{1}{C_{2}}=1$.

return to the original steady state but shifted horizontally as seen in the figure) analogous to the single Kuramoto-Sivashinsky case (see Smyrlis \& Papageorgiou 1990). Note that due to the scale of the horizontal axis the bursts appear to contain fast oscillations but on closer inspection typical times between oscillations are of the order of approximately $10^{3}$ time units, implying that the slow time-scale assumption remains valid.

Extensive numerical experiments (recall that $\nu=0.015$ is fixed) were carried out to produce the attractors given in table 2 and labeled as regions $I-V$ (these results were obtained by computing the most attracting solutions according to our initial-value problem). In region $I$ where $\zeta$ is non-zero but sufficiently small (i.e. $\zeta \lesssim 0.34415 \times 10^{-3}$ ), the dynamics are chaotic and the homoclinic burst behaviour found when $\zeta=0$ is lost. This was confirmed numerically for values of $\zeta$ as small as $4 \times 10^{-5}$. Typical results are shown in figure 9 for $\zeta=0.3162 \times 10^{-3}$; the absence of time intervals supporting steadystate solutions is evident from the figure, and the dynamics are chaotic. We find that the advective term introduced when $\zeta \neq 0$, extends the elliptic and Majda-Pego regions (due to the flux terms) in the $x-t$ plane, and destroys the appearance of steady-state attractors. These regions can be seen in panel (b) of figure 9 .

As $\zeta$ is increased further the chaotic attractor gives way to the time-periodic travelling wave attractor $I I$ (the solutions at large times are time-periodic travelling waves with 
$(a)$

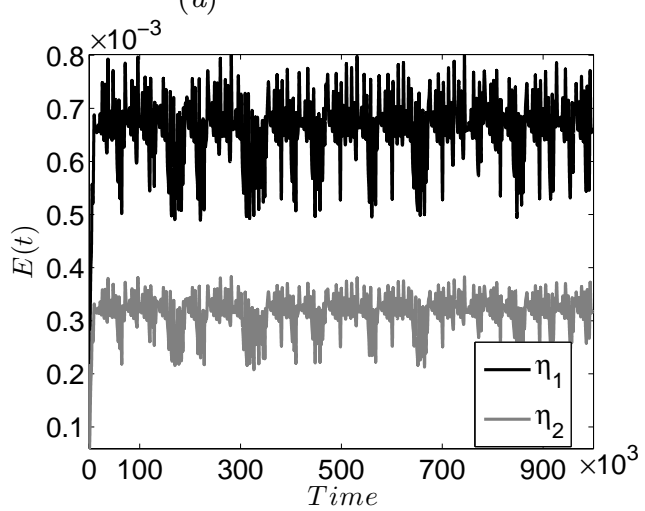

(b)

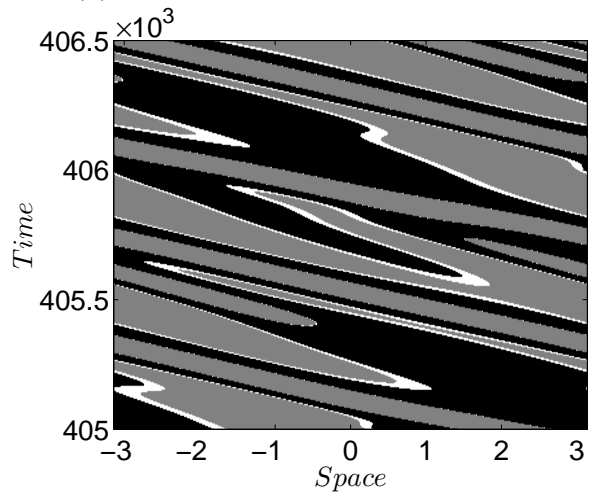

FIGURE 9. Chaotic interfacial oscillations for the non-symmetric system (3.12) when the inertia-associated matrix $s_{i j}$ is given by (4.5), $\zeta=0.3162 \times 10^{-3}, \nu=0.015$ and $\frac{1}{C_{1}}=\frac{1}{C_{2}}=1$. Panel $(a)$, the evolution of the energy; panel $(b)$, the spatiotemporal evolution of underlying instability regions: where black-shaded regions correspond to complex eigenvalues, white-shaded regions to Majda-Pego instability and gray-shaded regions indicate hyperbolicity.

periods depending on the value of $\zeta$ ). The dynamics inside region $I I$ are intricate and appear to follow a period-halving bifurcation scenario until a critical value of $\zeta$ is reached, after which a period-doubling cascade takes place and leads to the chaotic dynamics of region $I I I$. As $\zeta$ increases further, another periodic-travelling wave window is found, region $I V$.

The time-period at the start of region $I V$ is large and as $\zeta$ increases we observe a period-halving bifurcation (inverse Feigenbaum) that eventually leads to the steady-state travelling waves of region $V$, similar to those found in the symmetric case in Section 4.1 where the inertial terms were absent. To illustrate the dynamics in region $I V$ we present the phase-plane of $\left(E_{1}(t), \dot{E}_{1}(t)\right)$ for four values of $\zeta$ (shown on figure 10) that show three successive period-halving bifurcations. The transition from time-periodic solutions to steady-state travelling waves is monotonic in the sense that the single phase plane loop illustrated in the bottom right panel of figure 10, shrinks to a single point heralding steady-state dynamics. We can conclude, therefore, that when the kinematic terms dominate (relatively large $\zeta$ ), chaotic or time-periodic dynamics are regularised into steadystate travelling wave pulses. Illustrative results are given in figure 11 for three different values of $\zeta=0.94868 \times 10^{-3}, 1.3 \times 10^{-3}, 1.6 \times 10^{-3}$. The solutions have been shifted horizontally (allowed due to Galilean invariance of the equations) for comparison purposes, and further analysis and computations of the large $\zeta$-limit will be considered elsewhere. This regularisation is caused by terms that provide linear instability (elliptic advective terms) as opposed to more familiar dispersive regularisations encountered in single Kuramoto-Sivashinsky equations (see Akrivis et al. 2012).

\section{Conclusions}

In this paper we considered the linear and nonlinear stability of stratified multilayer flows in channels driven by gravity and horizontal pressure gradients. Fairly general physical systems have been considered in the case of three layer flows in channels characterized by immiscible fluids of different viscosities and densities. In addition, our models allow the underlying basic states to have different thicknesses for each liquid layer and in particular they support scenarios where less viscous fluids are occupying thicker or 

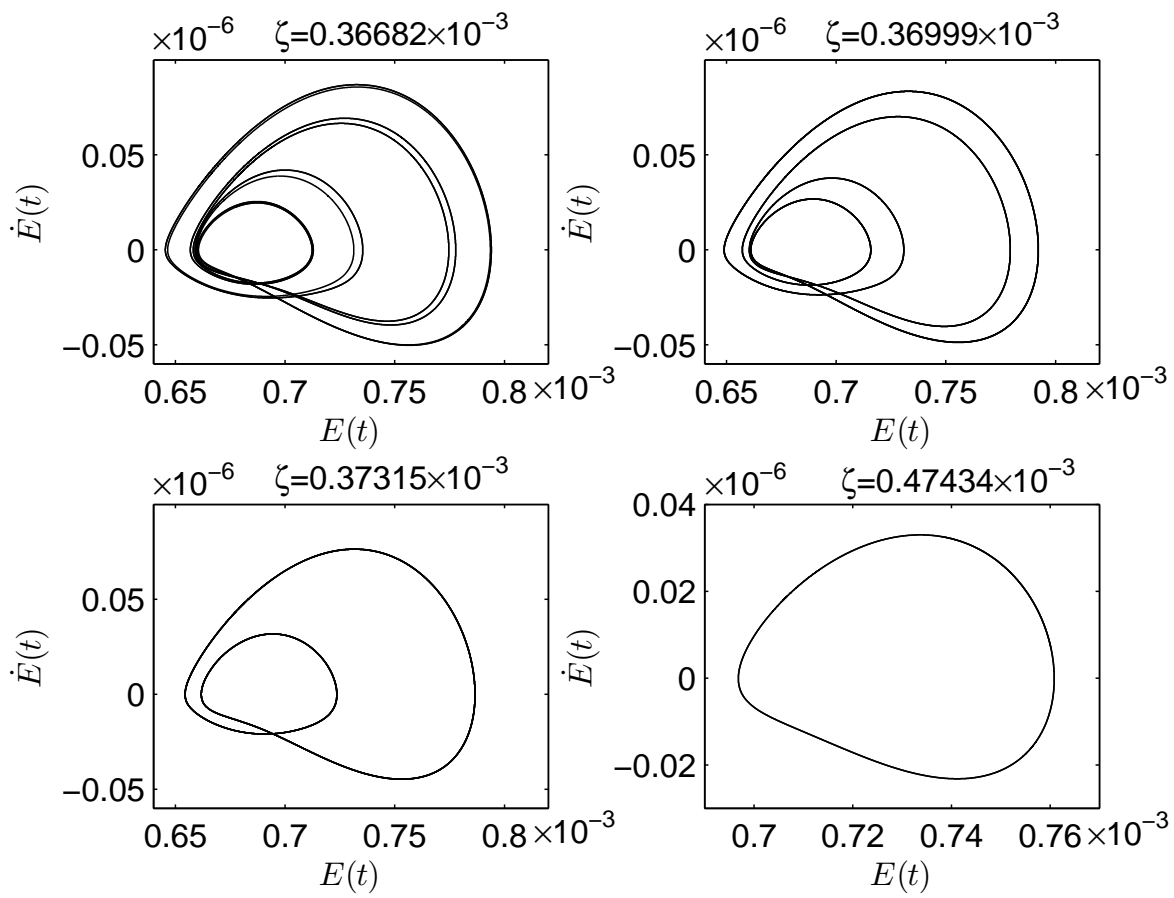

Figure 10. Phase planes of $\left(E_{1}(t), \dot{E}_{1}(t)\right)$ in region $I V$ as $\zeta$ increases - values shown on the figure. Three successive period-halvings are shown as we move from the upper left panel to the lower left one, clockwise. The dynamics transition from a period- 8 solution ( 8 turns in the phase plane), to a period-4, -2 and -1 solution. At larger $\zeta$ time-periodicity gives way to steady-state travelling waves - the phase plane shrinks to a point.

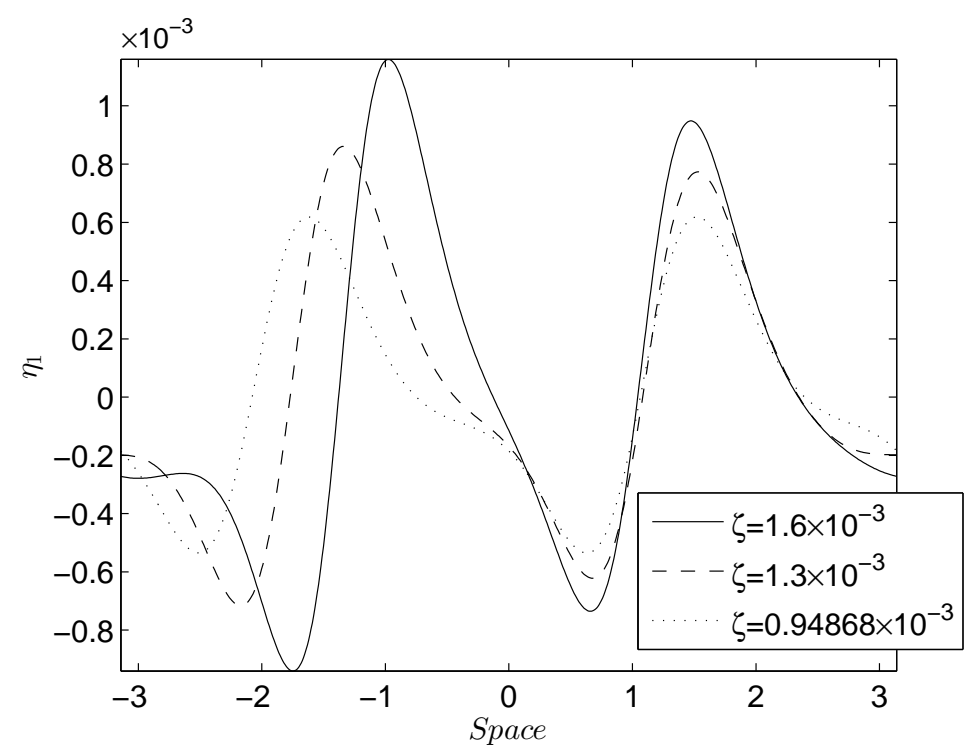

FIGURE 11. Emergence of steady-state travelling wave pulses as the values of parameter $\zeta$ increased considerably for fixed $\nu=0.015$ and $\frac{1}{C_{1}}=\frac{1}{C_{2}}=1$. 


$\begin{array}{ccc}\text { Attractor } & \zeta & \text { Description } \\ I & >0 & \text { chaotic oscillations } \\ I I & \gtrsim 0.34415 \times 10^{-3} & \text { periodic travelling waves } \\ I I I & \gtrsim 0.34848 \times 10^{-3} & \text { chaotic oscillations } \\ I V & \gtrsim 0.36651 \times 10^{-3} & \text { periodic travelling waves } \\ V & \gtrsim 0.72416 \times 10^{-3} & \text { steady-state travelling waves }\end{array}$

TABLE 2. Overview of the solutions of the kinematically modified cKS system when the inertia-associated matrix $s_{i j}$ is given by (4.5), $\nu=0.015$ and $\frac{1}{C_{1}}=\frac{1}{C_{2}}=1$; The values of $\zeta$ denote an approximate lower bound for the described attractor.

thinner liquid layers, respectively. Ten dimensionless parameters emerge (two of each of viscosity, density, surface tension, and basic state thickness ratios, a Reynolds number and a Capillary number), making the problem challenging and physically rich. Our focus has been in making analytical progress by deriving asymptotically correct coupled systems of weakly nonlinear evolution equations that can be used to understand the dynamics. The derivation of the models is carried out in two stages. First, a system of nonlinear equations is derived valid for interfacial deformations that are long compared to typical undisturbed layer thicknesses; in addition, the wave amplitudes scale with the layer thicknesses (or equivalently the channel height). This system is worked out to second order in the slenderness parameter $\delta$ in order to regularize the leading order system that typically encounters singularities in finite time (if a solution exists for small times at all) that violate the long-wave approximation. The resulting equations are a system extension to the Benney-type equations obtained for flows with a single interface, and this has a crucial effect on carrying out consistent asymptotic approximations. Since the parameter $\delta$ in the regularized system cannot be scaled out of the problem, we proceed with a weakly nonlinear analysis to produce canonical models (without small parameters present) that retain nonlinearities and all the different stabilising and destabilising physical mechanisms of the problem. The main technical issue involved in correctly carrying out a weakly nonlinear expansion is the fact that the linearised (about the flat states) leading order Benney system has unequal eigenvalues in general, so that there are two unequal speeds of wave propagation. In single interface problems such terms are removed by a Galilean transformation with the weakly nonlinear models following by balancing first order terms with the nonlinearity. In the present case we require the eigenvalues to be real and equal so that a Galilean transformation can be applied to the system simultaneously. This condition defines, in general, a six-parameter family of admissible basic states and we have reduced the solution space, for relative simplicity, by taking the three fluid densities to be equal, leaving us with the two viscosity and two depth ratios as the controlling parameters (the general case can be analysed in a directly analogous manner). Basic states satisfying this condition (the resulting parameters are such as to give what is known as an umbilic degenerate point for the unregularised system of conservation laws) were explored in detail and two canonical cases arise. The first case, termed symmetric (see figure 2(a) and section 3.1), emerges by perturbing parameters and carrying out a weakly nonlinear analysis about a flow with all layers having equal viscosity and with the thicknesses of the top and bottom layers being equal. The resulting evolution equations (3.6) are inertialess (the viscosity stratification instability enters at higher order) but contain nonlinear fluxes of mixed hyperbolic-elliptic type. The second canonical case, termed non-symmetric, perturbs about basic states with different layer 
viscosities and thickness ratios and the analysis is described in section 3.2 (see figure 2(b) for a typical base flow profile), resulting in the system of evolution equations (3.12). In this case inertia enters and was found to destabilise the flow for viscosity and thickness ratios explored here. The nonlinear flux functions are again of mixed type and consequently enhance the rate of energy input that comes from the inertial terms (note that surface tension provides short-wave stabilisation rendering the equations well-posed).

The symmetric inertialess systems generically evolve to produce nonlinear travelling wave coherent structures like the ones depicted in figure 5 . These nonlinear structures can emerge in one of two ways: (i) from infinitessimally small initial perturbations in the case when the system is linearly unstable with respect to the flat states $\left(\eta_{1}=\eta_{2}=0\right)$ - here the instability is either due to Majda-Pego instability or the "alpha"-effect (see Section 3.1); (ii) from initial disturbances of amplitudes above a threshold value in the case when the flat states are linearly stable to all wavelengths. We note that the required threshold amplitudes are moderate - for example in the computations depicting this phenomenon in figure 6 the initial energy required is approximately 1.164 which is equivalent to a scaled amplitude 0.657 of an equivalent monochromatic sinusoidal initial condition. We have established that the reasons for this are due to the underlying hyperbolic-elliptic transitions and Majda-Pego instabilities found in our systems of multilayer equations. An important conclusion that can be drawn from these findings is that in the case of three stratified layer flow (we surmise that systems with additional interfaces will behave analogously), linear stability analysis is a necessary but not sufficient condition for the emergence of non-trivial nonlinear coherent structures.

The presence of inertia found in the non-symmetric case can provide a destabilising mechanism that enriches the dynamics of the system and in general enables complex chaotic dynamics to emerge in many parameter regimes. Note that such mechanisms can arise even at zero Reynolds numbers due to capillary and Marangoni instabilities, (see for example Papageorgiou et al. 1990; Kalogirou et al. 2012). Illustrative examples have been given in the absence $(\zeta=0)$ and presence $(\zeta \neq 0)$ of the linear kinematic terms in the flux function of (3.12); we consider two cases (following unslaved and slaved dynamics respectively) characterised by strongly chaotic dynamics, and chaotic homoclinic bursts, respectively, when $\zeta=0$ (see figures 7 and 8 ). The kinematic terms (measured through $\zeta$ in our models) can promote hyperbolic-elliptic transitions as well as MajdaPego types of instabilities, and comprise an additional source of instability even in the presence of "negative diffusion" caused by the inertia (see the positive definite second order diffusion matrix $S$ in (3.12)). We have carried out extensive numerical experiments to determine the effect of increasing $\zeta$ on the dynamics, and have found that solutions which are time-periodic or chaotic when $\zeta$ is zero or small, ultimately become regularised into steady-state travelling waves at sufficiently large $\zeta$. In particular for the slaved dynamics case, table 2 summarises these findings and two interesting dynamical phenomena emerge: (i) the behaviour as $\zeta$ increases is non-monotonic, i.e. there are alternating windows supporting chaotic dynamics and time-periodic solutions; (ii) the transition from chaotic dynamics to the ultimate nonlinear travelling waves that emerge at "large" $\zeta$ (in the example of table 2 these waves appear at $\zeta \geq 0.72416 \times 10^{-3}$, approximately), takes place through a reverse Feigenbaum cascade via a sequence of period-halving bifurcations. Our numerical findings are consistent with the Feigenbaum universal theory but a significant amount of additional simulations is required to establish this. We also emphasize that the regularisation of the underlying complex dynamics by enhancing the kinematic instability terms is a novel regularisation phenomenon that is quite distinct from the more familiar dispersive regularisations found in single nonlinear evolution equations (e.g. the dispersively modified Kuramoto-Sivashinsky equation - see (Akrivis et al. 
2012)). In the present problem large $\zeta$ enables the system to promote the kinematic terms compared to the inertial ones thus pushing the system towards the canonical symmetric case equations that produce travelling-wave states at large times.

\section{Appendix A. The extra condition for the velocities $\tilde{w}_{i}^{(0)}$}

Using (2.40), the integral expression for the leading order normal velocity $\tilde{w}_{3}^{(0)}$ in the bottom fluid layer 3 reads

$$
\tilde{w}_{3}^{(0)}=-\int_{0}^{y} \tilde{u}_{3 \xi}^{(0)} d y
$$

while in the top layer 1 we have

$$
\tilde{w}_{1}^{(0)}=-\int_{h_{2}}^{y} \tilde{u}_{1 \xi}^{(0)} d y+c_{w 1} .
$$

The no-slip condition at $y=1$ gives

$$
c_{w 1}=\int_{h_{2}}^{1} \tilde{u}_{1 \xi}^{(0)} d y .
$$

The normal velocity in the middle layer 2 is given by

$$
\tilde{w}_{2}^{(0)}=-\int_{h_{3}}^{y} \tilde{u}_{2 \xi}^{(0)} d y+c_{w 2},
$$

and continuity of velocities at the lower-most interface $y=h_{3}$ gives

$$
c_{w 2}=-\int_{0}^{h_{3}} \tilde{u}_{3 \xi}^{(0)} d y \text {. }
$$

Consequently, the remaining extra condition corresponding to continuity of normal velocities at $y=h_{2}$ reads

$$
\int_{0}^{h_{3}} \tilde{u}_{3 \xi}^{(0)} d y+\int_{h_{3}}^{h_{2}} \tilde{u}_{2 \xi}^{(0)} d y+\int_{h_{2}}^{1} \tilde{u}_{1 \xi}^{(0)} d y=0 .
$$

This condition can also be obtained by differentiating the overall flow-rate (2.33) with respect to $\xi$ and using the Liebniz formula since the limits of integration are functions of $\xi$.

ESP acknowledges the support of a Roth Ph.D. scholarship by the Department of Mathematics, Imperial College London. DTP was partly supported by NSF grant DMS0707339, and GAP was supported by EPSRC grants EP/J009636/1 and EP/H034587/1.

\section{REFERENCES}

Akrivis, G, Papageorgiou, D.T. \& Smyrlis, Y.-S. 2009 Linearly implicit methods for a semilinear parabolic system arising in two-phase flows. IMA J. Numer. Anal. 31, 299-321. 
Akrivis, G., Papageorgiou, D.T. \& Smyrlis, Y.-S. 2012 Computational study of the dispersively modified Kuramoto-Sivashinsky equation. SIAM J. Sci. Comput. 34, A792-A813.

Beebe, D. J., Mensing, G. A. \& Walker, G. M. 2002 Physics and applications of microfluidics in biology. Annu. Rev. Biomed. Eng. 4, 261-286.

Bell, J. B., Trangenstein, J. A. \& Shubin, G.R. 1986 Conservation laws of mixed type describing three-phase flow in porous media. SIAM J. Appl. Math. 46, 1000-1017.

Benjamin, T.B. 1957 Wave formation in laminar flow down an inclined plane. J. Fluid Mech. 2, 554-574.

Benney, D.J. 1966 Long waves in liquid films. J. Math. Phys. 45, 150-155.

CAnIC, S. \& Plohr, B. J. 1995 Shock wave admissibility for quadratic conservation laws. J. Differ. Equations 118, 293-335.

Chen, K.P. 1993 Wave formation in the gravity driven low Reynolds number flow of two liquid films down an inclined plane. Phys. Fluids A 5, 3038-3048.

Chen, K.P. 1995 Interfacial instabilities in stratified shear flows involving multiple viscous and viscoelastic fluids. Appl. Mech. Rev. 48, 763-776.

Chumakova, L., Menzaque, F.E., Milewski, P.A., Rosales, R.R., Tabak, E.G. \& Turner, C.V. 2009 Shear Instability for Stratified Hydrostatic Flows. Comm. Pure App. Math. 62, 183-197.

Cole, J.D. \& Cook, L.P. 1996 Transonic Aerodynamics. Elsevier Science Publishers B.V.

Evans, L.C. 2010 Partial Differential Equations. AMS.

Figeys, D.L \& Pinto, D. 2000 Lab-on-a-Chip: A revolution in biological and medical sciences. Anal. Chem. 72, 330 A-335 A.

GAO, P. \& LU, X.-Y. 2008 Mechanism of the long-wave inertialess instability of a two-layer film flow. J. Fluid Mech. 608, 379-391.

Hibara, A., Tokeshi, M., Uchiyama, K., Hisamoto, H. \& Kitamori, T. 2001 Integrated multilayer flow system on a microchip. Anal. Sci. 17, 89-93.

Hooper, A. P. \& Grimshaw, R. 1985 Nonlinear instability at the interface between two viscous fluids. Phys. Fluids 28, 37-45.

Jiang, W.Y., Helenbrook, B.T., Lin, S.P. \& Weinstein, S.J. 2005 Low-Reynolds-number instabilities in three-layer flow down an inclined wall. J. Fluid Mech. 539, 387-416.

Joseph, D.D. \& Renardy, Y.Y. 1991 Fundamentals of Two-Fluid Dynamics, Part I: Mathematical Theory and Applications. Springer-Verlag.

Kalogirou, A., Papageorgiou, D.T. \& Smyrlis, Y.-S. 2012 Surfactant destabilization and non-linear phenomena in two-fluid shear flows at small Reynolds numbers. IMA J. Appl. Math. 77, 351-360.

KAO, T.W. 1968 Inertialess instability of a two-layer liquid film flow. J. Fluid Mech. 33, 561.

KLIAKHANDLER, I. 1999 Long interfacial waves in multilayer thin films and coupled KuramotoSivashinsky equations. J. Fluid Mech. 391, 45-65.

Kliakhandler, I. \& Sivashinsky, G. 1995 Kinetic alpha effect in viiscosity stratified creeping flows. Phys. Fluids 7, 1866-1871.

KliakhandleR, I. \& Sivashinsky, G. 1996 Inertial effects and long waves in multilayer plane Poiseuille flows. Phys. Scripta T67, 90-95.

Kogan, M.N. 1961 On magnetohydrodynamic flows of mixed type. PMM 25, 132-137.

LI, C.-H. 1969 Instability of three-layer viscous stratified flow. Phys. Fluids 12, 2473-2481.

Loewenherz, D. S. \& LaWrence, C. J. 1989 The effect of viscosity stratification on the instability of a free surface flow at low Reynolds number. Phys. Fluids A 1, 1689.

Lovick, J. \& ANGeli, P. 2004 Experimental studies on the dual continuous flow pattern in oil-water flows. Int. J. Multiphas. Flow 30, $139-157$.

Majda, A. \& PEgo, R.L. 1985 Stable viscosity matrices for systems of conservation laws. $J$. Differ. Equations 56, 229-262.

Milewski, P., Tabak, E., Turner, C., Rosales, C. \& Menzaque, F. 2004 Nonlinear stability of two-layer flows. Commun. Math. Sci. 2, 427-442.

Nordberg, M. E. \& Winter, H. H. 1988 Fully developed multilayer polymer flows in slits and annuli. Polym. Eng. Sci. 28, 444-452.

Papageorgiou, D.T. \& Orellana, O. 1998 Study of cylindrical jet breakup using onedimensional approximations of the Euler equations. SIAM J. Appl. Math. 59, 286-317. 
Papageorgiou, D. T., Maldarelli, C. \& Rumschitzki, D. S. 1990 Nonlinear interfacial stability of core-annular film flows. Phys. Fluids A 2, 340-352.

Peters, G. R. \& Canic, S. 2000 On the oscillatory solutions in hyperbolic conservation laws. Nonlinear Aanal.-Real. 1, 287-314.

Polsin, K.A. \& Choueiri, E.Y. 2002 A passive propellant feeding mechanism for micropropulsion using capillarity. In 38th AIAA/ASME/SAE/ASEE Joint Propulsion Conference and Exhibit.

Pozrikidis, C. 2004 Instability of multi-layer channel and film flows. Adv. Appl. Mech. 40, 179 $-239$.

Pumir, A., Manneville, P. \& Pomeau, Y. 1983 On solitary waves running down an inclined plane. J. Fluid Mech. 135, 27-50.

Rosenau, R.J., Oron, A. \& Hyman, J. M. 1992 Bounded and unbounded patterns of the Benney equation. Phys. Fluids A 4, 1102.

Schaeffer, D.-G. \& Shearer, M. 1987 The classification of $2 \times 2$ systems of non-strictly hyperbolic conservation laws, with application to oil recovery. Comm. Pure App. Math. 40, $141-178$.

Shlang, T. \& Sivashinsky, G. I. 1985 Irregular wavy flow due to viscous stratification. J. Physique 46, 863-866.

Skurtys, O. \& Aguilera, J. 2008 Applications of microfluidic devices in food engineering. Food Biophys. 3, 1-15.

Slemrod, M. 1983 Admissibility criteria for propagating phase boundaries in a van der Waals fluid. Arch. Rational Mech. 81, 301-315.

Smyrlis, Y.-S. \& PAPAgeorgiou, D.T. 1990 Computational study of chaotic and ordered solutions for the Kuramoto-Sivashinsky equation. ICASE Rep. 96-12, pp. 1-32.

Smyrlis, Y.-S. \& PAPAgeorgiou, D.T. 1991 Computational study of chaotic and ordered solutions for the Kuramoto-Sivashinsky equation. Proc. Natl. Acad. Sci. USA 88, pp. 1112911132.

SooHoo, J.R. \& WALker, G.M. 2009 Microfluidic aqueous two phase system for leukocyte concentration from whole blood. Biomed. Microdevices 11, 323-329.

Surmeian, M., Slyadnev, M. N., Hisamoto, H., Hibara, A., Uchiyama, K. \& Kitamori, T. 2002 Three-layer flow membrane system on a microchip for investigation of molecular transport. Anal. Chem. 74, 2014-2020.

Tilley, B. S., Davis, S. H. \& BAnkoff, S. G. 1994a Linear stability theory of two-layer fluid flow in an inclined channel. Phys. Fluids 6, 3906-3922.

Tilley, B. S., Davis, S. H. \& Bankoff, S. G. $1994 b$ Nonlinear long-wave stability of superposed fluids in an inclined channel. J. Fluid Mech. 277, 55-83.

Weinstein, S. J. \& Ruschak, K. J. 2004 Coating flows. Annu. Rev. Fluid Mech. 36, 29-53.

YIH, C.-S. 1963 Stability of liquid flow down an inclined plane. Phys. Fluids 6, 321-335.

Yin, ChiA-Shun 1967 Instability due to viscosity stratification. J. Fluid Mech. 27, 337-352.

Zauderer, E. 2006 Partial Differential Equations of Applied Mathematics. John Wiley \& Sons. 